$\frac{40 / 10191}{1019}$

2

PREPARED FOR THE U.S. DEPARTMENT OF ENERGY, UNDER CONTRACT DE-AC02-76-CHO-3073

PPPL-2783

UC-420

\title{
OVERVIEW OF TFTR TRANSPORT STUDIES
}

BY

R.J. HAWRYLUK, et.al.

October 1991

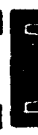

PRINCETON

plasma physics

lagofatoGY

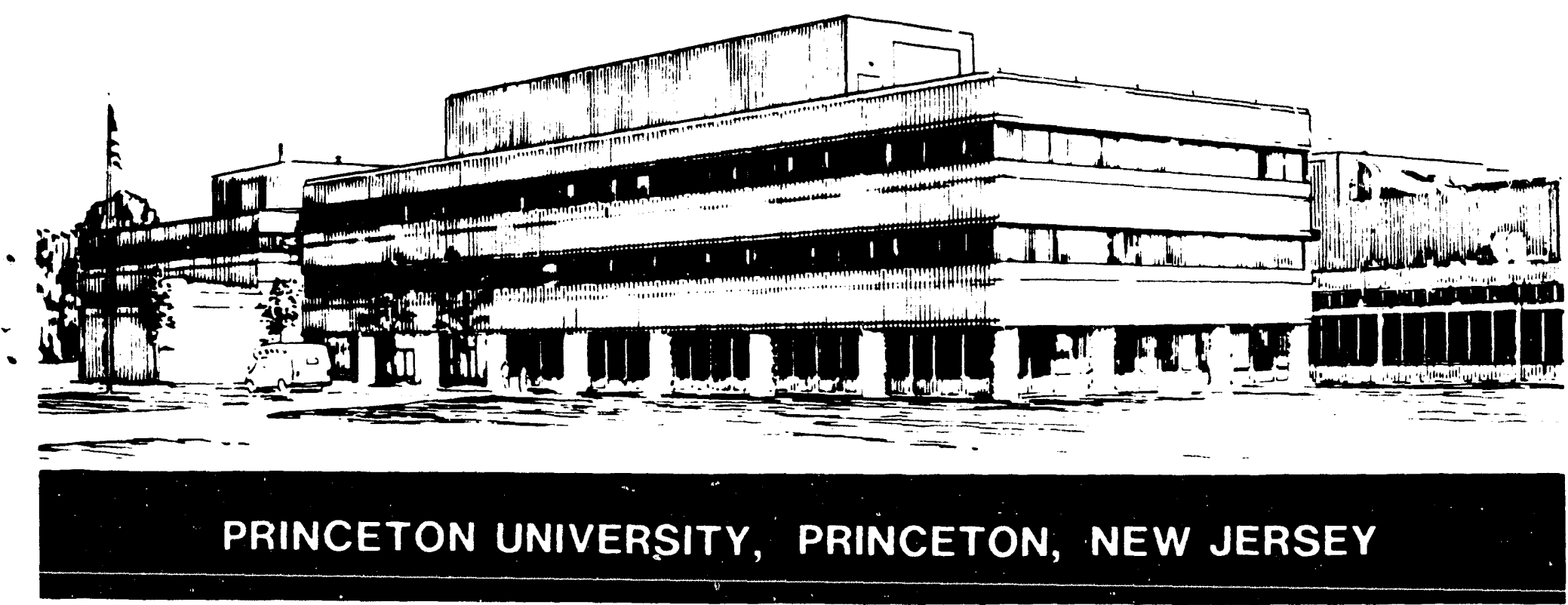

DHETROUTION OF THIS DOCUMENT $18 \mathrm{UN}$ 


\section{NOTICE}

This resport was prepared as an account of work sponsored by an agency of the United States Government. Neither the United States Government nor any agency thereof, nor any of their employees, makes any warranty, express or implied, or assumes any legal liability or responsibility for the accuracy, completeness, or usefulness of any information, apparatus, product, or process disclosed, or represents that its use would not infringe privately owned rights. Reference herein to any specific commercial produce, process, or service by trade name, trademark, manufacturer, or otherwise, does not necessarily constitute or imply its endorsement, recommendation, or favoring by the United States Government or any agency thereof. The views and opinions of authors expressed herein do not necessarily state or reflect those of the United States Government or any agency thereof.

\section{NOTICE}

This report has been reproduced directly from the best available copy.

Available to DOE and DOE contractors from the:

Office of Scientific and Technical Information

$$
\text { P.O. Box } 62
$$

Oak Ridge, TN 37831 ;

Prices available from (615) 576-8401.

Available to the public from the:

National Technical Information Service

U.S. Department of Commerce

5285 Port Royal Road

Springfield, Virginia 22161

703-487-4650 
R. J. HAwryiUk, V. Arunasalam, C. W. Barnesl, M. Beer, M. Bell, R. Bell,

H. Biglazi, M. Bitter, R. Boivin, N. L. Bretz, R. Budny, C. E. Bush 2, C. 2. Cheng,

$\therefore$ K. Chu, S. A. Cohen, S. Cowley, P. C. Efthimion, R. J. Eonck ${ }^{3}$, E. Eredrickson,

H. ?. Furth, R. J. Goldston, G. Greene, B. Grek, L. R. Grisham, G. Hammett,

iN. Heidbrink4, K. W. H111, J. Hosea, R. A. Hulge, H. Hsuan, A. Janos, D. Jassby,

E. I. Jobes, D. W. Johnson, L. C. Johnson, J. Kesner ${ }^{5}, C$. Kieras-Phillips,

S. J. Kilpatrick, H. Kugel, P. H. La Marche, B. LeBlanc, D. M. Manos,

J. K. Mansfield, E. S. Marmar ${ }^{5}$ E. Mazzucaco, M. P. McCarthy, M. Mauel ${ }^{6}$,

J. C. McCune, K. M. McGuire, D. M. Meade, S. S. Medley, D. R. Mikkelsen,

O. Monticello, R. Motley, D. Mueller, Y. Nagayama7, G. A. Navrat116, R. Nazikian,

O. K. Owens, H. Park, W. Park, S. Paul, F. Perkins, S. Pitcher , A. T. Ramsey,

M. H. Redi, G. Rewoldt, D. Roberts3, A. L. Roquemore, P. H. Rutherford,

S. Sabbagh 6 , G. Schilling, J. Schivell, G. L. Schmidt, S. D. Scote, J. Snipes 5 ,

. Stevens, B. C. Stzatton, W. Stodiek, E. Synakowski, X. Takases, W. Tang,

G. Taylor, J. Terrys, J. R. Timberlake, H. H. Towner, M. Ulrickson, S. von Goeler,

R. Wieland, M. Will1ams, J. R. Wilson, K. L. Wong, M. Yamada, S. Yoshikawa,

K. M. Young, M. C. Zarnstorff, and S. J. Zweben

\author{
Plasma Physics Laboratory, Princeton University \\ P.O. Box 451 Princeton, New Jersey 08543 USA \\ LLos Alamos National Laboratory, New Mexico \\ 2Oak Ridge National Laboratory, Oak Ridge, IN \\ University of Wisconsin, Madison, WI \\ 4University of Callfornia, Irvine, CA \\ 5 Massachusetts Institute of Technology, Cambridge, MA \\ ${ }^{6}$ Columbia University, New York, ary \\ TUniversity of Tokyo, Tokyo, Japan \\ BCanadian Eusion Euels Technology Project. Toronto, Canada
}

\title{
ABSTRACT
}

A zeview of TETR plasma transpez studies is presented. Parallel transport and the confinement of suprathermal ions are found to be relatively well described by theory. Cross-field transport of the thermal plasma, however, is anomalous with the momentum diffusivity being comparable to the ion thermal diffusivity and larger than the electron thermal diffusivity in neutral beam heated discharges. Perturbative experiments have studied non-linear dependencies in the transport coefficients and examined the role of possible non-local phenomena. The underlying turbulence has seen studied using microwave scactering, beam emission spectroscopy and microwave ceflectometry over a much broader range in $x \perp$ than previously possible. Results indical:e the existence of large-wavelength fluctuations correlated with enhanced zransport.

MHD instabilities set important operational constraines. However, by modifying the cutzent profile using current ramp-down techniques, it has been possible to extend she operating regime to higher values of both $e \beta_{p}$ and normalized $\beta_{\tau}$. In addition, the interaction of MHD fluctuations with fast ions, of potential relevance to $\alpha$ particle confinement in D-T plasmas, has been investigated. The installation of cazbon-carbon composito tiles and improvements in wall conditioning, in particular the use of 11 pellet infection to reduce the carbon recycling, continue to be important in the improvement of plasma performance.

\section{INTRODUCTION}

: zansport studies have been a principal focus of the research activities on TETR for wo main reasons. First, in order to achieve TETR's research objectives during the D-T phase, a regime of enhanced confinement ("supershors") compared with L-mode has had to be developed to enhance the plasma reactivity (Strachan et al., 2987; Hawryluk et al., 1987; Bell et al., 2988; and Meade ot al., 1990) second, results from transport studies have important implications for the design and eventual operation of the next generation of devices (BPX and ITER). During the past three 
yeazs, transport studles have been given cenewed emphasis in :he U.S. program with the establishment o . national Transport Task Force (TTF) (iallen et al., 1990). comprehensive zeviews of transport issues have been recently published by the rTE (Boozer et al., 1990; wootron ot al., 1990; Burrell et al., 1990; Houlberg et al., 1990; Kaye et al., 1990; and Stambaugh at al., 1990). On TFn, detailed transpozt studies have been performed in a broad range of operacing cond: tions lohmic, L-mode, supershot, pellet enhanced, and $\mathrm{H}$-model to characterize and unclerstand the transport mechanisms with the goal of improving plasma pertormance. As will be discussed below, these experiments have supported the predictions of som theoretical models. However, in other areas, some of the underlying assumptions conmonly used hove been brought into question. This paper will provide a summary of the principal transport studies conducted on TETR and will highlight those experimencal results which a comprehengive theory of plasma transport would have to describe.

This paper will begin by reviewing parallel transport and the confinement of suprathermal ions, both of which are relatively well described by theory. The more complex topic of cross-field transport of the thermal components will then be discussed. Rerturbative experiments will be described which test virious theoretical models and address the role of non-linear or non-local mechanisms. In addition, studies of confinement scaling in terms of non-dimensional parameters have been conducted to form a physics-based approach to predict the performance of future machines. Studies of the underlying surbulence and the role of MHD instabilities on zansport and confinement will be summarized. The paper will conclude with a brief discussion of the development of wall condltioning techniques, which continue to play a key role in plasma confinement experiments.

A description of che machine hardware capabilities for the experiments descrtbed here has been given by Meade ot al. (1990). TETR has a circular cross-section plasma with minor radius, a, variable from $0.4 \mathrm{~m}$ to $0.96 \mathrm{~m}$ and major radius, Rp, variable from $2.1 \mathrm{~m}$ to $3.1 \mathrm{~m}$. The coroidal field system is operated at the full field rating of $B_{T}-5.2 T$ and plasma currents $I_{p}$ up to $3 M A$ have been achieved. The neutral beam system has injected a total power of up to $P_{N B I}-33 m$. The ICH systems have coupled inco the plasma up to PICH - 6.3MW. The 1 imiter system for TETR consigts of an axisymmetric inner-wall bumper limitgr and two carbon/carbon composite poloidal rings on the outer cross-section to protect the ICH antennas. In easly 1990, the graphite tiles in the high heat flux regions of the inner-wall bumper limiter were replaced with carbon-carbon fiber reinforsed tiles. TFTR has two pellet injectors. An eight barrel $D_{2}$ pellet injector, developed and built by oak Ridge National Laboratory, produces pellets of $3,3.5$ and $4 \mathrm{~mm}$ diameter at speeds of $-1.5 \mathrm{~km} / \mathrm{sec}$. A two barrel lithlum of carbon pellet injector developed. built and operated by Massachusetts Institute of Technology, produces $2 \mathrm{~mm}$ pellets at $0.8 \mathrm{~km} / \mathrm{sec}$ for fueling, diagnostlc, and wall coaring experiments.

The experiments which will be described principally involve auxiliary heated discharges in the supershot, $L$-mode and $H$-mode regimes. Plasma parameters extend up $=0 I_{1}(0)-35 \mathrm{keV}, I_{0}(0)-12 \mathrm{keV}, n_{0}(0)-1.2 \times 10^{20} \mathrm{~m}^{-3}$ producing $D-D$ neutron rates up to $5 \times 1016 / \mathrm{sec}$ in supershots. The fusion parameter $n_{0}(0) T_{\varepsilon} T_{1}(0)$ in supershot plasmas has reached values of $4.4 \times 10^{20} \mathrm{~m}^{-3}$.suc.kev. Hot-ion plasmas combining peaked density profiles with the edge characteristics of H-mode plasmas have been produced in circular cross-section discharges yielding $n_{0}(0) \tau_{E} T_{1}(0)$ values characteristic of supershots (Bush et al., 1990). With deuterium-pellet injection, central dengities of $5 \times 10^{20} \mathrm{~m}^{-3}$ have been achieved enabling the study of high density discharges as well. 


\section{PARALLEL TRANSPORT}

The plasma response to the inductive electric field and other sources of current is analyzed using the neoclassical (classical spitzer resistivity plus trapped-particle effects) Ohm's law obcained from parallel electron momentum balance (Hirshman and Sigmar, 1981). On TETR, Zarnstorff et al. (1990a) found the plasma surface voltage in onmically-heated discharges to be in good agreement with the predictions of neoclassical sesistivity and in disagraement with the predictions of classical cesistivity when the measured temperature profile, zetf, equilibrium parameters and the calculated bootstrap current ( 55 of $I_{p}$ ) were taken into account. This agreement is in contrast with the observed cross-field electron transport which is typically much larger ( $>100$ ) than neoclassical predictions. In these experiments, the collision frequency $v_{0}^{*}(r-a / 2)$ was varied from 0.07 to 2 , enabling a detailed test to be made of the predictions of the neoclassical resistivity over the transition from the banand into the placeau regime.

Juring moderate current ramps ( $d I_{p} / d t \leq 1 M A / s$ ) both the surface voltage and the location of the $q$ - 1 surface are well predicted on the basis of neoclassical resistivity. Fredrickson et al. (1987) perfomed current penetration studies with higher current ramping rates, up to $3.2 \mathrm{MA} / \mathrm{s}$. In these cases, bursts of MHD activity were observed during the ramp as $q$ (a) crossed rational values of $5,4.5$ and 4 . A small distuption was observed at the $q(a)$ - 4 crossing which was accompanied by al drop in the election temperature. The resulting increase in the calculated resistivity due to the rapid change in the electron temperature enhanced che calculated surrent penetration, and resulted in good agreement with the experiment without having to postulate anomalous current diffusion. Current diffusion has not been studied dufing the initial $200 \mathrm{~ms}$ of the discharge, when the current ramp rate is faster (dI $/ d t \geq 5 M A / s)$.

Curzents driven by density and remperature gradients (bootstrap current) and by unthermalized beam ions due to unbalanced (co- and counter-tangential) injection are also well predicted by neoclassical theory. These have been extensively studied by zarnstorff et al. (1987, 1988 a) and scote et al. (1989) who found good agreement with neoclassical predictions using the time-dependent analysis code TRANSP (Hawryluk, 1980; Goldston ot al., 1981). In Eig. 1, the measured surface voltages in two discharges are compared with predictions of neoclassical theory for nearly balanced injection (Fig. La) in which the bootserap current is predicted to be dominant (up to 65), and with co-injected noutral beams only (Elg. ib) in which the beam-driven current dominates. In evaluating the co- only injection discharge, it was necessary to include the effects of the measured toroidal plasma rotation on beam depositior and slowing-down of the beam ions to obtain agreement. The agreement with theory in these experimerts has given strong support to the models presently used to calculate the bootstrap and the beam driven current in future devices such as BPX and ITER.

\section{FAST ION CONEINEMENT}

Cross-field tzansport of fast lons (beam injected lons, multi-MeV fusion products and minority ions heated by $I C H)$ comparable to that for the thermal plasma would have substantial implications not only for the interpretation of present experiments sut also sor reactor requirements. This topic has been extensively studied on TETR. An elegant experiment by Radeztsky et al. (2988), using tangential neutral beams injected into the edge of a discharge and a tangentially viewing detector to measure the charge-exchange neutral flux, placed an upper bound on the diffusivity of passing fast ions $(30-90 \mathrm{keV})$ of $\mathrm{D}_{\text {past }}<0.05 \mathrm{~m}^{2} / \mathrm{s}$. Recently, short $(-20 \mathrm{~ms})$ pulses of injected beam ions were used to study the confinement of both passing and trapped 

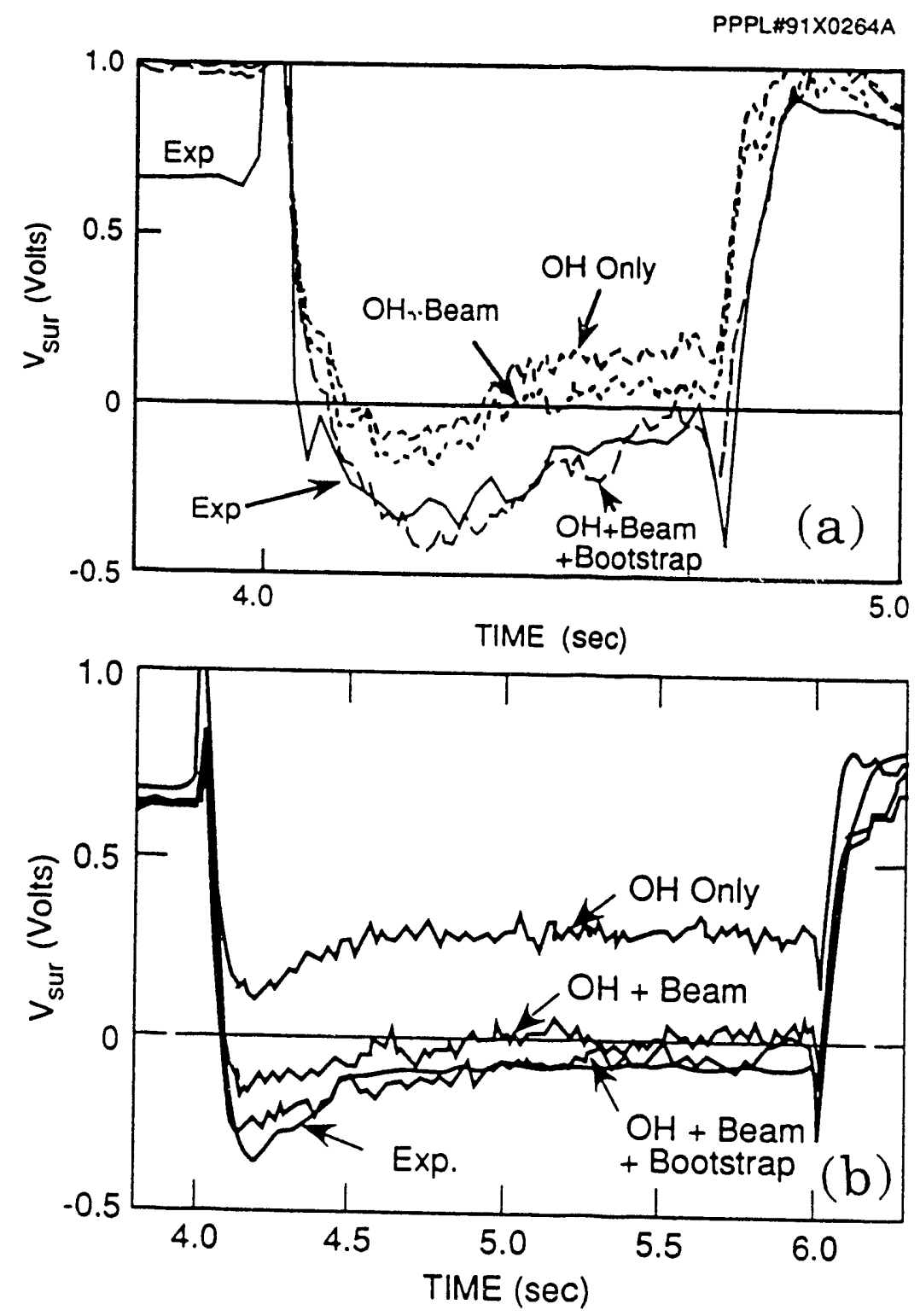

Fig. 1 a) The measured surface voltage in a discharge with nearly balanced injection compared with TRANSP simulations including driven currents and with simulations excluding the bootstrap current and without the beam-driven current. ( $I_{p}=1.0 \mathrm{MA}, \mathrm{P}_{\mathrm{NBI}}=11 \mathrm{MW}$ ) b) Same comparison for a discharge heated by coinjection. ( $\left.I_{p}=1.0 \mathrm{MA}, P_{N B}:=11 \mathrm{MW}, V_{Q}=8 \times 105 \mathrm{~m} / \mathrm{s}\right)$ 
particles (90keV) (Scott ot al., 1990; Heidbrink et al., 1991). The decay rate of the fusion neution production following the pulse was found to agree well with sheoretical calculations based on classical slowing down and no radial diffusion. in upper bound on the radial transport was inferred to be $0.1 \mathrm{~m}^{2} / \mathrm{s}$. This conclusion was also supported by measurements of the neutron emission profile and measurements of cnarge-exchange neutrals along two vertically viewing chords.

Measurements of the ICH-generated energetic ions in TFTR indicate that these trapped ions experience very little radial transport compared to the strong anomalous iransport of the thermal plasma. Figure 2 shows the measured flux of $100 \mathrm{keV}$ hydrogen to a charge-exchange detector viewing vertically through the plasma at a major radius of $3.23 \mathrm{~m}\left(=R_{p}+0.65 \mathrm{a}\right.$, with $R_{p}=2.61 \mathrm{~m}$ and $a=0.96 \mathrm{~m}$ for this plasma), as a function of the cyclotron resonance layer position. Since the detector is riewing perpendicular to the magnetic field, it sees particles which have chargeexchanged at their banana tips. The $I C H$ accelerates the hydrogen primarily in $v_{\perp}$ chus producing a very lazge signal when the cesonance layer is directly over the jetector. Eigure 2 presents the predicted flux (normalized at $R=3.2 \mathrm{~m}$ ) from a comprehensive bounce-averaged quasilinear Fokker-Planck code (Hammetr, 1986). This code is coupled to a full-wave ICH deposition code (Smithe et a1., 1987) which alculates the propagation and deposition of the fast wave. The measurements can be matched with $D_{\text {case }}=0.05 \mathrm{~m}^{2} / \mathrm{s}$, which is larger than the neoclassical prediction, $-0.01 \mathrm{~m}^{2} / \mathrm{s}$, but roughly consistent with the transpcrt expected to be caused by coroidal sield ripple. Nevertheless, the fast lons experience very little crosssield transport relative to the electron thermal diffusivity, $x_{0}$, which was $1 \mathrm{~m}^{2} / \mathrm{s}$.

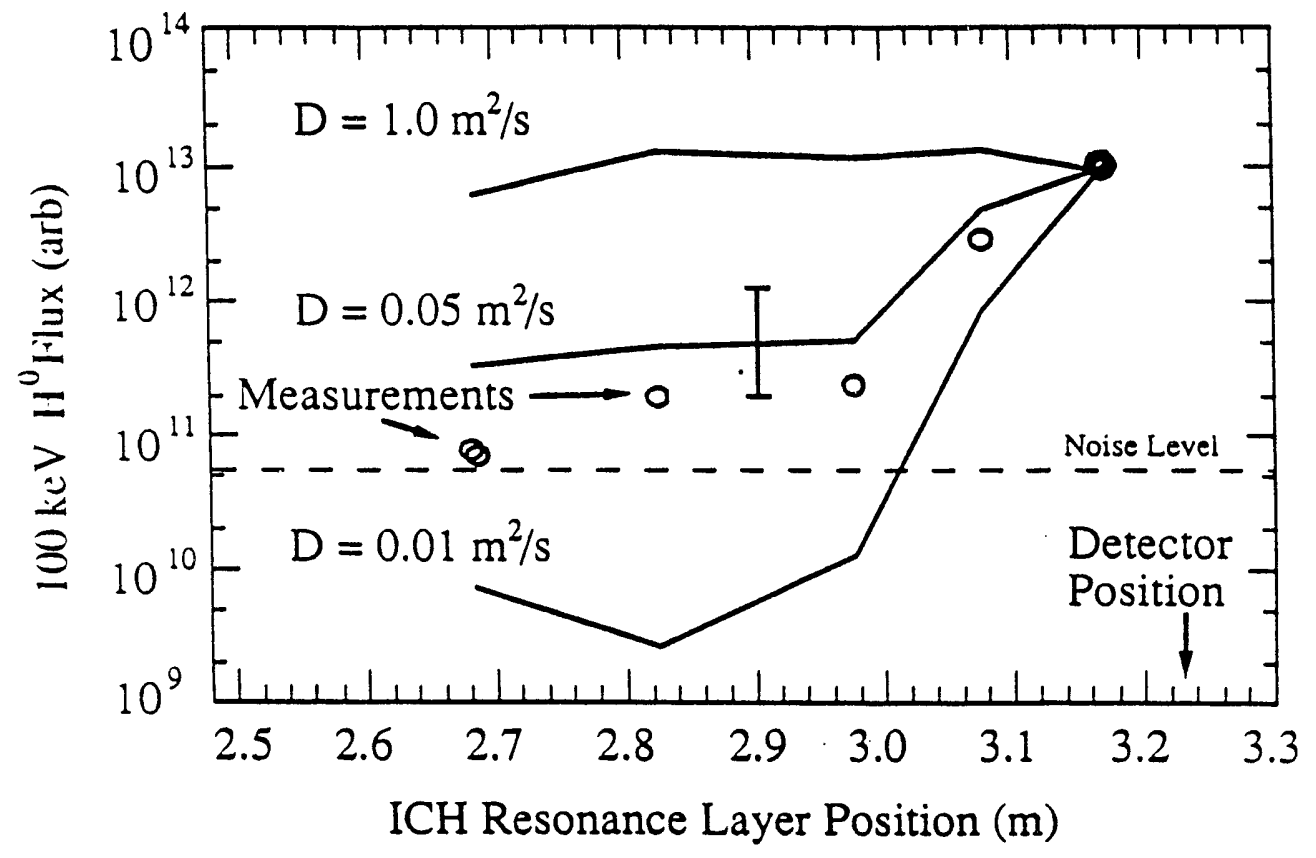

Eig. 2 Charge-exchange measurements of lookev resonant minority ions as a function of the location of the ICH resonance layer compared with the results of FokkerPlanck calculations for different levels of radial eransport. 
Fast tritons produced by $d(d, p) t$ reactions at $2 \mathrm{MeV}$, which slow down primarily by electzon drag, may undergo subsequent $t(d, n) \alpha$ reactions to produce 14 MeV neutrons. The triton burnup fraction (in steady-state, the zatio of the D-I neutzon to the D-D reutzon ratel is a very sensitive function of triton slowing down and confinement. The ratio of the measured triton burnup fraction to the expected fraction is less than ore and tends to decrease (to about 0.3 ) in discharges with long slowing-down : imes (Scote et al., 1990 C). The discrepancy is consistent with a radial diffusion sefficient of $-0.1 \mathrm{~m}^{2} / \mathrm{s}$. Radial diffusion of fast fusion products at this race in she burning plasmas of BPX or ITER would not be a severe loss, due to the relatively snort thermalization times for fusion alpha particles.

Direce measurements of the loss of MeV D-D fusion products (tritons and protons) near the botcom of the vacuum vessel wall $\left(45^{\circ}-90^{\circ}\right.$ below the outer midplane) and just below the midplane are shown in Figs. 3 and 4 . The loss of fusion products away from the midplane is consistent with the expected first-orbit loss in MHD guiescent discharges and is observed to decrease with increasing cursent (zweben et a1., 1990). (The effects of MHD on fast particle behavior will be discussed in sec. ;III). The cross-field diffusivity of passing particles above $0.5 \mathrm{MeV}$ has been shown to be Dest $\leq 0.1 \mathrm{~m}^{2} / \mathrm{s}$ (2weben et al., 1991). A midplane probe has been used :o gtudy loss associaced with toroldal field stochastic ripple diffusion (Boivin

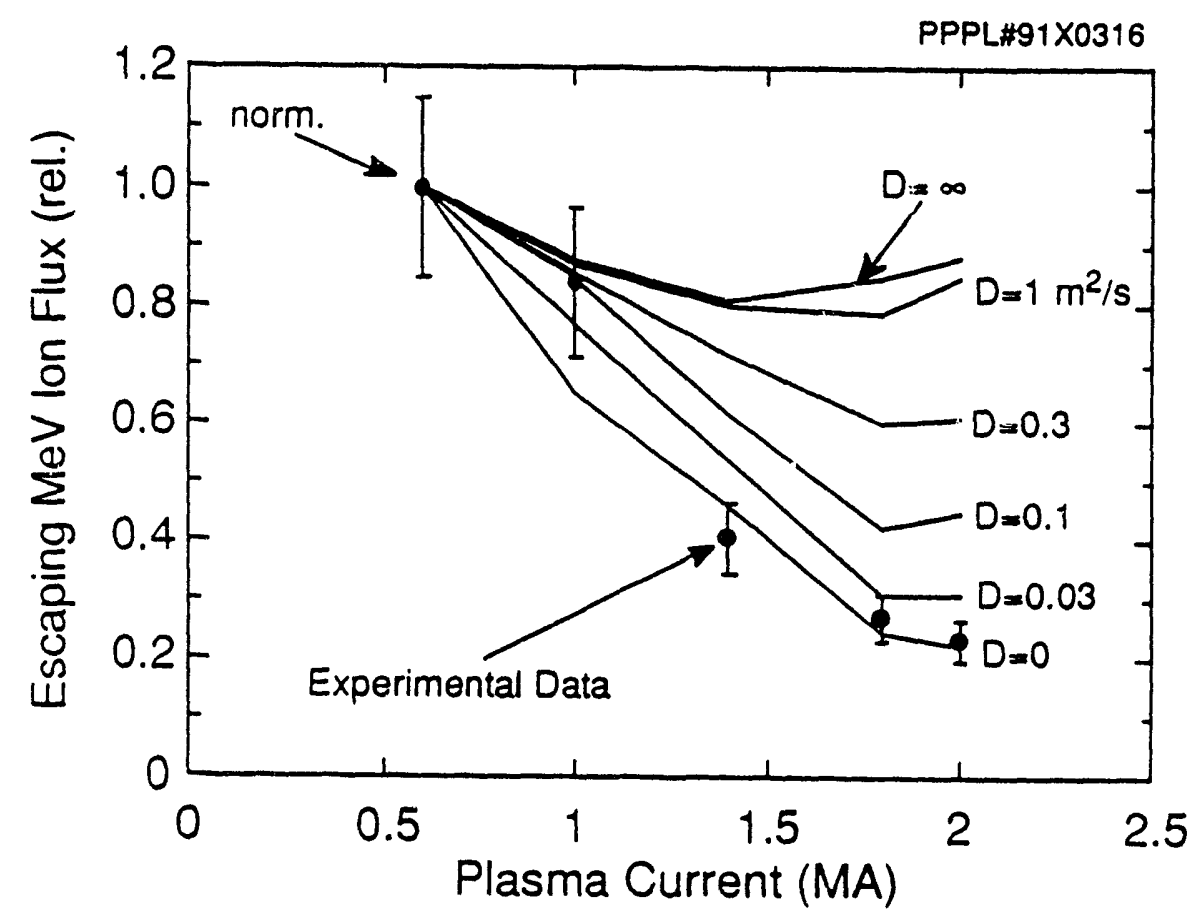

E.g. 3 Ratio of the measured ion loss to the total neutron flux versus plasma current for a probe mounted at the bottom of the vessel. The calculated curves include losses due to bad orbits and lncreasing levels of the radial diffusivity. The observed decrease in ion loss with plasma currene is consistent with the predicted loss of lons on bad orbits only, lndicating little cross-field transport. ( $R_{P}-2.60 \mathrm{~m}, \mathrm{~B}_{\mathrm{T}}=3.7 \mathrm{~T}, \mathrm{P}_{\mathrm{HBI}}=5-12 \mathrm{MW}$ ) 


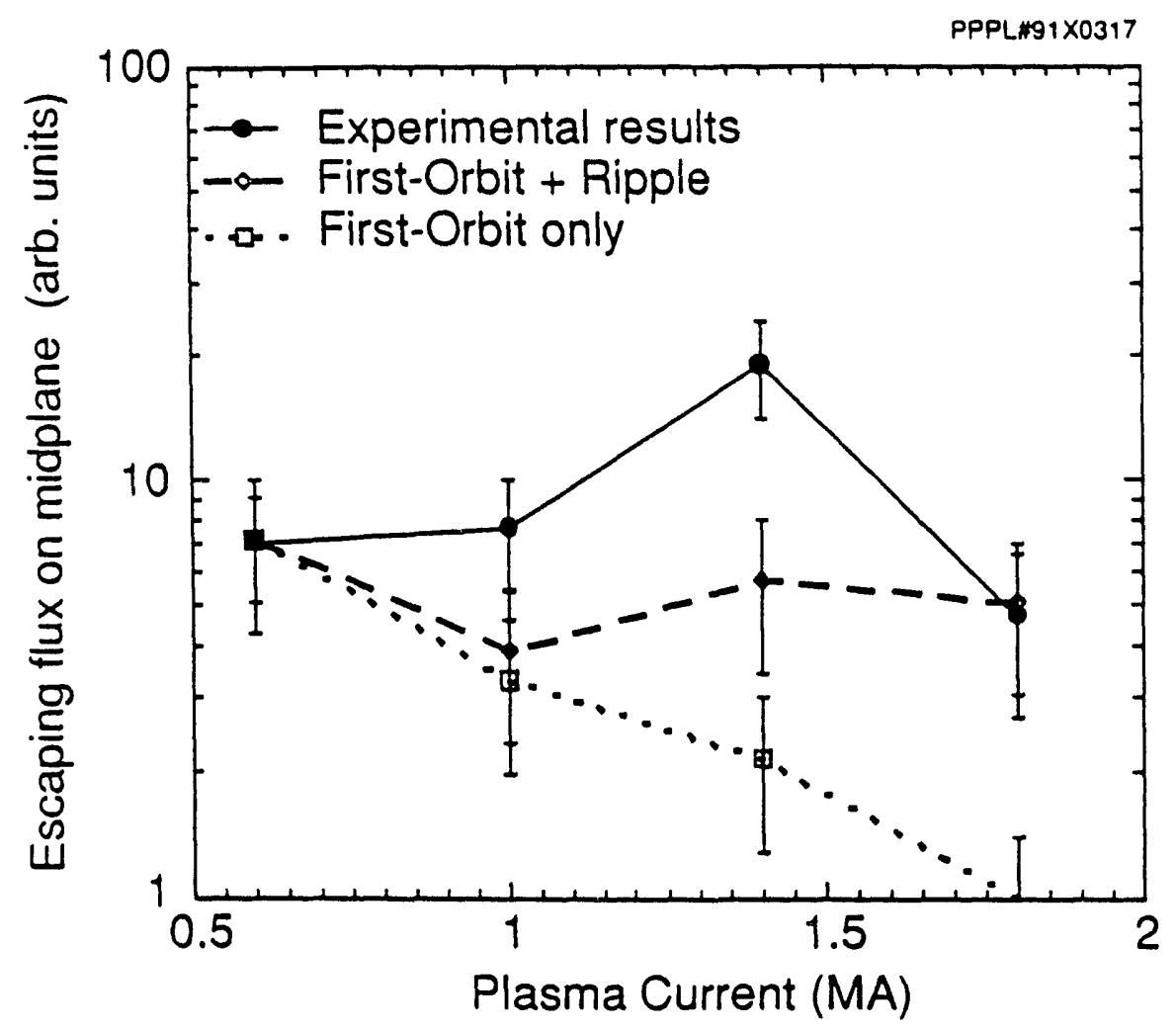

E1g. 4 Ratio of the measured ion loss to the total neutron flux versus plasma current for a probe mounced near the midplane for the same scan shown in Fig. 3. The predicted losses due to bad orbits and toroidal field ripple stochastic diffusion are shown.

et al., 1991). This is a potentially important transport mechanism because, when the ripple stzength exceeds about 0.1 , the bounce point of the energetic lon diffuses vertically untll the lon hits the wall. Initial modeling of the re ripple diffusion and the experimental measurements are in reasonable accord, as shown in Fig. 4 for the same data set as in Fig. 3, confirming the presence of the losses predicted by Goldston, white, and Boozer (1981). The difference between the model and the experimental results is believed to be due to the location of the detector, which is placed outside the RF limiter radius, but well inside the first wall. The derector can sample part of the confined ion population, explaining qualltatively cho additional flux at medium currents.

The lack of strong anomalous transport for the fast ions, as found in all of these measurements, is believed to be the result of two effects. The dominant reason is that, in the absence of high-frequency MHD, e.g., Alfvenic fluctuations such as the -oroidal Alfven eigenmode (TAE) or kinetic ballooning modes, there are no resonant modes for the fast ions in these experiments (Efthimion et al., 1989; Biglari and chen, 1991). This favorable cizcunstance may not be satisfied in the D-T phase of TETR operacion, where a-particles are expected to destabilize both taE and kinetic ballooning modes, as will be discussed in Sec. VIII. A secondary factor is that even if the resonance condition could be satisfled, orbit-averaging effects, which 
occur when the ion banana width is largo compared to the turbulence correlation length, ceduce the driving Eorce (Mynlck and Duvall, 1989).

\section{CROSS-FIELD TRANSPORT}

Cross-field transport studles have been conducted over a large paramerer range $10.3<I_{p}<3 M A, I<B_{T}<5 T, 0.4<a<0.9 \mathrm{~m}, 2.1<R_{p}<3.1 \mathrm{~m}, P_{N B I} \leq 33 M W$, and $P_{I C H} \leq 6.3 \mathrm{MW}$ ) and in many operating regimes (L-mode, supershot, H-mode, and with pellet injectiont. Eurthermore, a sophisticated set of profile diagnosties with good spatial and temporal sesolution has enabled us to conduct perturbation experiments to test various theoretical models.

The results from the steady-state transport studies, which have been described in detail by scott et al. (1989, 1990a-c), Zarnstorfet al. (2989a,b) and Efthimion et al. (1991b), will be briefly summarlzed. The effective lon heat diffusivity, $x_{1}^{e::}=-q_{1} / n_{1} \nabla T_{1}$, is large compared with the neoclassical value (Eonck et al., 1989), a result similar to the DIII observations by Groebner et al. (1985). The electron, $\chi_{\theta}^{e l t}$, and momentum $\chi_{\phi}^{\theta}$ diffusivities are also much larger than

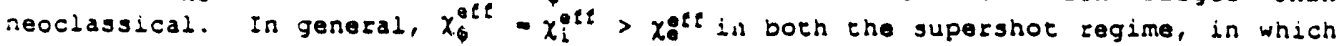
the density profile is highly peaked on axis, and in the L-mode regime with broad dengity profiles. This is shown in Fig. 5. As the density profile peakedness $n_{e}(0) /\left\langle n_{e}\right\rangle$ is varied from 1.5 to 3 largely by changes in wall recycling, the resulting discharge conditions vary continuously from the L-mode to the supershot regime with variations of $\tau_{\varepsilon} / \tau_{\varepsilon}^{L}$ Erom 1 to 3 , where $\tau_{\varepsilon}^{L}$ is the prediction of $L$-mode scaling (Goldston, 1984). During thls process, $x_{1}^{\text {tlt }}$ is reduced by a factor up to eight within 5 < a/2 whereas $x^{2} t$ is reduced by a factor of only about two. The particle diffusivity (ignoring possible pinches) is of the same order as $x g^{\text {ft }}$ in the plasma core $x e^{\prime l t}-(2-2.5) \times D e f t$ at $r / a-0.27$ and decreases as the density profile peakedness increases. In the core of high current supershor discharges, both $x_{1}^{a f t}$ and $x^{\text {tf }}$ decrease with increasing neutral beam power despite the tact that the usual driving terms ( $I_{1}, T_{0}, n_{0}, \beta \ldots$ ) all increase.

Ion energy transport becomes so low in the core of supershot plasmas that heat conduction is negligible compared to convection. This contrasts with the usual situation in L-mode discharges wharo conduction dominates. The strong central Eveling by the beam particles provides a well defined source of particles and heat, which in sceady-state is balanced by an outward flux. The ratios Qe/ I. To and $\partial_{1} / \Gamma_{1} I_{1}$ (wheze $Q$ and $\Gamma$ aze the respective hear and particle fluxes) yield upper bounds on the minimum value of the ratio of heat eransport by convection to particle flux for each species (2arnsejeff ot al., 1988b; 1989a). It is tound that is supershors, the upper bound on the minimum value of $Q_{1} / \Gamma_{1} I_{1}$ is $-3 / 2$ for balanced and councer-beam infection, though for highly-rotaring plasmas with co-only injection the upper bound is $s 1$. The upper bound on the minimum value of $Q_{0} / \Gamma_{0} T_{0}$ is typically -2 independent of the direction of beam injection. The values of these upper bound catios are found to increase with minor radius, corresponding to an increase of conduction compared with convection, assuming that the multiplier on the convection term does not change with minor radius. Comparing the measured ratio of $2(\varepsilon) / \Gamma(E) I(E)$ with theory appears to be a promiging techilique for esaluating different models of anomalous transport.

The relationsnips between $x_{e t} x_{1}^{e t}, x_{0}^{e t}$ and the pirticle diffusivities, as well as the upper bound on the convective heat tlux, place significant constraints on theoretical transport models. The observation that $x_{\text {eft }}<x_{1}^{\text {et }}-x_{0}^{\text {eft }}$ is consistent with the predictions for electrostatically-driven transport. it is inconsistent with $V_{\|} a$ transport being the predominant mechanism throughout cho plasma, alshough an electromagnecic model in which the turbulence is intermitcent or the 

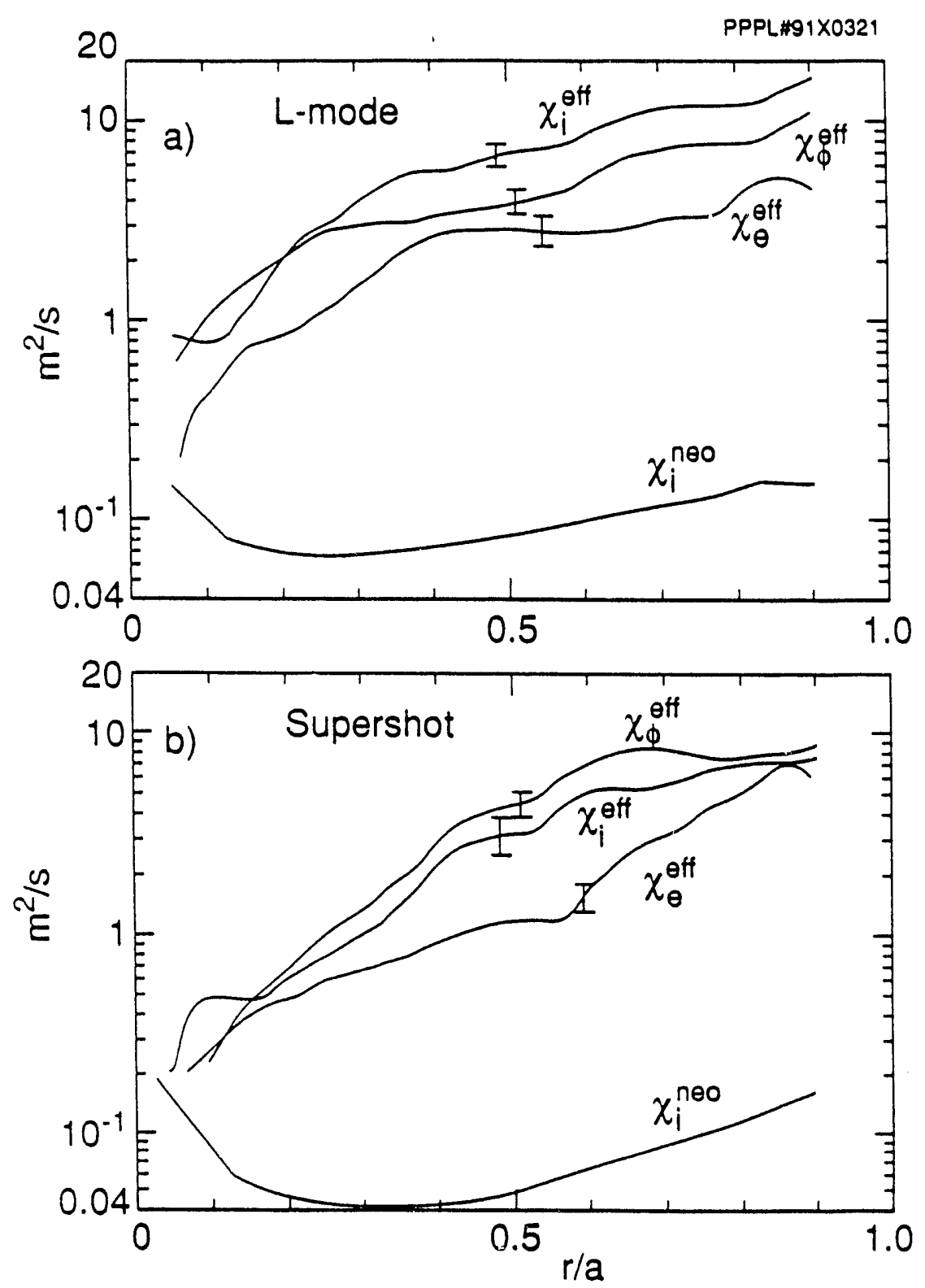

Fig. 5 vartations of $\chi^{\text {ett, }}, \chi_{1}^{\text {elt }}$ and $\chi_{\phi}^{\text {ett }}$ and $\chi_{1}^{\text {neo }}$ with minor radius for a) an L-mode plasma and b) a supershot. For both discharges $R_{p}=2.45 \mathrm{~m}, a-0.8 \mathrm{~m}, I_{p}=1.3 \mathrm{MA}, P_{N B I}=21 \mathrm{MW}$.

Eransport along stochastic fleld lines occurs in some reglons where the surfaces are destroyed, while other intact regions provide an effective transport barrier, may still be possible. However, for models in which eransport along stochastic magnetic Ejelds determine the transport, the minimum ratio of $Q_{0} / \Gamma_{0} T_{0}$ should be of order $\sqrt{m_{1} / m_{e}}$ whereas for the electrostatic driven transport $Q_{0} / \Gamma_{0} T_{\bullet}-1.5$. Once agaln the data suggests thal electrostatic turbulence is dominant, at least in the case of supersnors. 
Analysis of momentum transport avoids some of the complications associated with the analysis of heat transport because the convective momentum loss is much less important and the ine-tia of electrons is not significant. In addition, it provides further ingight into another important plasma property. The central rotation speed turing neurzal beam heating modulated at $5 \mathrm{~Hz}$ (Scott et al., 1987) ghowed a clear modulation with centrally-weighted heating. During modulated edge-weighted hectjng, in which the beam deposition profile peaked at $r / a=0.6$, the central rotation velocicy showed a simple secular rise. This behavior is consiscent with a simple diffusive momentum transport mechanism (without a momentum pinch) with a $x_{\phi}^{\text {etf }}$ that is a Eacror of -2 higher during central heating than during edge neating. The observation that the final rotation speed attalned after 3 beam cycles of edgeheating was fully $2 / 3$ of that attained during central-heating implies that diffusive zzansport of momentum must dominate in this experiment over all local momentum damping mechanisms (e.g. ripple, charge-exchange) as well as any outwardly directed momentum flows (i.e. a positive pinch velocity).

The diffusive nature of momentum transport is also appazent in the evolution of the velocity profile $v_{b}(R)$ from an edge-peaked to a flat proflle during the first $500 \mathrm{~ms}$ of constant edge heating as shown in Elg. 6 (Takase, et al., 1991). Similar to the modulated-beam data, the measured velocity profile can be reproduced without introducing a momentum pinch. Interestingly, the velocity profile "fills in" from the edge in an apparently diffusive manner, while the inferred (neoclassical) electrostatic potential increases secularly with time, due to the increasing velocity. This indicates that momentum transport cannot be driven by a relaxation of the electrostatic potential, as would be expected for example from ripple or electromagnetic turbulence coupled to the wall. Slmilarly, we orserve no changes in local temperatures and no changes in local heat-transport coefflcients in rotating or guasi-stationary L-mode plasmas 1 the rotation speed is perturbed by transiently altering the mix of co- and counter-injecting beams (at constant $P_{N B I}$ ).

The vagiation of $t_{E}$ with aspect ratio $(2.8<R / a<8.0)$ has been studied by Grisham et al. (1990, 1991) in L-mode plasmas. The corfinement time deduced from the diamagnetic measurement is in reasonable agreement with the ITER power scaling relationship (Yushmanov et al., 1990) multiplied by a factor 0.8 - 0.85. However, the confinement deduced from detailed kinetic profile measurements yields a more favorable dependence on aspect ratio, $\left.\tau_{\Sigma} \propto R^{(1,1}-1.8\right) a^{0}$, closer to the scaling of Goldston (1904). In this experiment, an especially interesting observation is tnat $x^{e f f}$ is betcer described as a function of the local inverse aspect ratio (e - r/R) than as a function of normalized minor radius (r/a). This data suggests that increasing inverse aspect ratio, rather than the local value of electron temperature or $a$, is responsible for the enhanced transport in the piasma periphery. The isotope dependence of cross-field transport was also studied in a series of ohmic and L-mode NBI discharges. The scaling of energy confinement with ion mass is found to be weaker than expected: for TETR $\tau_{E} \propto A^{0.24}$ whereas for ITER-P $\tau_{E} \propto A^{0.5}$. Due to the difficulty in changing from deuterium to hydrogen in TFTR, there was a significant concentration (25 - 55) of deuterium in the nominally hydrogen discharges that has been taken into account in deriving the exponent for the mass.

\section{PERTURBATION EXRERIMENTS}

The radial variation of particle and heat fluxes (and the resuleing vartation of the density and cemperature profiles) has been explored in a series of perturbation experiments. Electron particle and thermal transport coefeiclents were measured during a controlled scan in which the plasma temperature was varied by a factor of 2 whilo the electron density, plasma current, and corcidal magnetic fleld were held constant (Efthimion et al., 1991a). The purpose was to determine 1 f there is any 


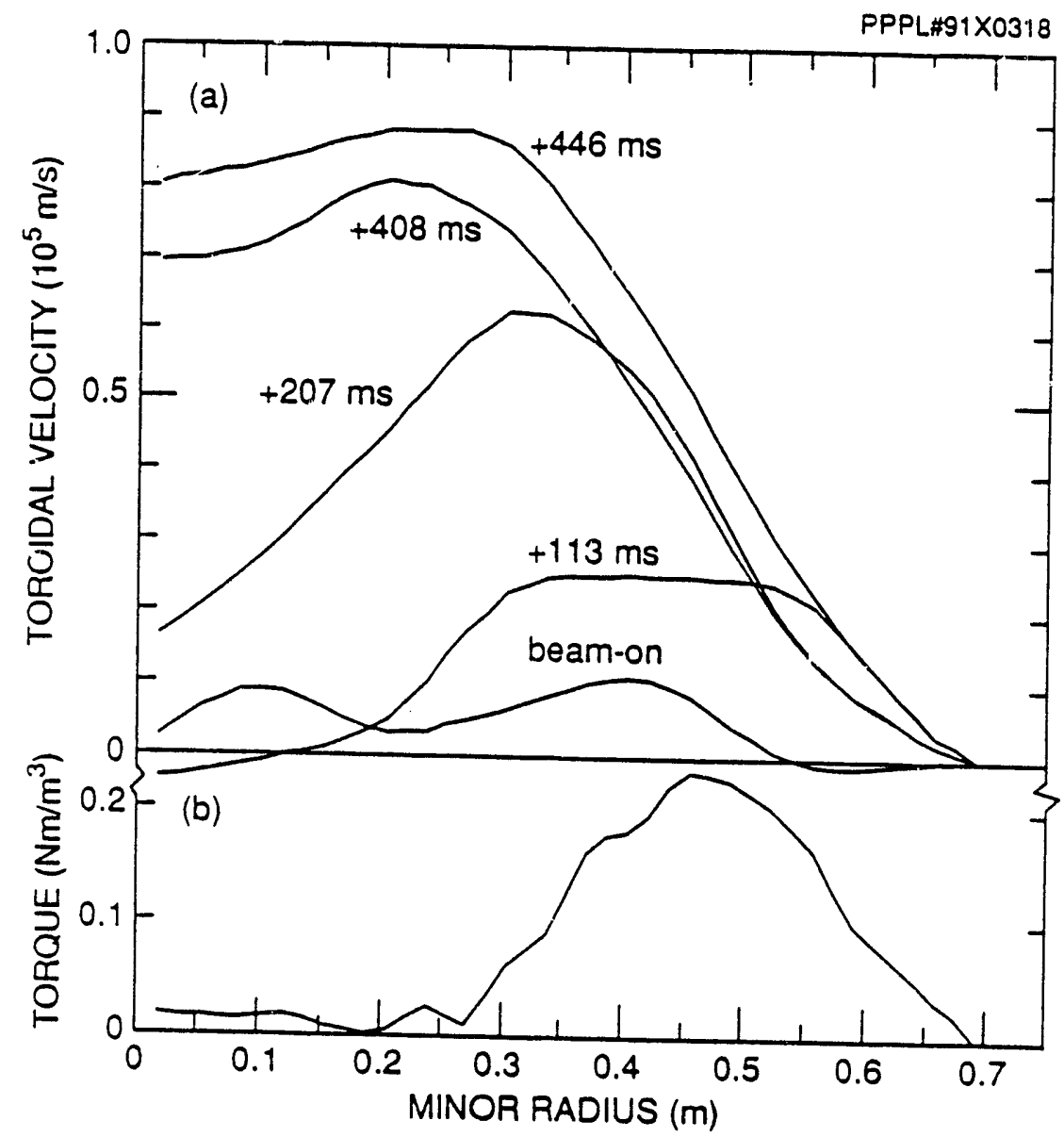

Eig. 6 Profiles of a) the toroidal rotation velocity at increasing times through the beam pulse and b) the toroidal torque from the injected neutral beam during an edge-heating experiment. $\left(R_{p}-2.3 \mathrm{~m}, B_{T}-5 T, I_{p}\right.$ 1.2MA, $\left.P_{\mathrm{NBI}}=1.1 \mathrm{MW}\right)$

explicit temperature dependence of electron transport in auxiliary heated plasmas. The particle transpozt was measured with a perturbative gas puffing technique. A small amount of gas is introduced at the edge during a steady-state beam-heated plasm and the time evolutions of the density profile and the particle sources are used to ascertain the particlo transport coeffictents. The analysis uses a general : Zux relationship that allows for nonlinear transport which is fitced to the experimental particle flux over the time of the perturbation at each plasma radius: $\delta \Gamma=\left(\partial \Gamma / \partial \nabla_{n}\right) \delta \nabla_{n}+(\partial \Gamma / \partial n) \delta n$ where the transport coefficients are in terms of these partial derivatives. Previously, Efthimion et al. (1989) analyzed the perturbed Elux with the assumption that $\Gamma=-D \nabla_{n}+\nabla n$ which fnherently assumed that $D$ and the plach velocity $\nabla$ are weak functions of $n$ and $\nabla_{n}$. This assumption has been found to ce incorrect. In the temperature scan, the electron themal diffusivity profile, $x^{\prime} t_{(f)}$, was obtained from equilibrium power balance analysis. rhe analysis of the 
particle and heat transport coefficients ylelds local cemperature dependencies varying as $T_{0} \alpha$ where $\alpha=1.5-2.5$. Quasilinear models of microinstability theory have predicted this observed temperature dependence (Rewoldt and Tang, 1990 ) for the coroidal drift mode destabilized by the combined influence of trapped electrons and ion temperature gradients. It should be noted that $\chi^{e l t} \times T^{b}$.5 would zesult in tE PNBI-0.6 which is approxinately consistent with the widely observed l-mode confinement power scaling.

Taking the partial derivatives of the particle fluxes $\partial \Gamma / \partial \nabla n$ and $\partial \Gamma / \partial n$ at each radius one can look for additional dependencies in the data. The two partial derivatives normalized to - temperature dependence vary with minor radius by factors of 30 to 50. There are few parameters that can fit the radial variations, and comply with the constraint $\partial / \partial n(\partial \Gamma / \partial \nabla n)-\partial / \partial \nabla n(\partial \Gamma / \partial n)$. The parameters that do satisfy the cross-partial constraint and the large variation in the normalized partial derivatives are the density and temperature scale lengths. The functional fits to the partial derivatives are integrated to obtain the form of the total particle flux. The best fits to the data are fluxes of tine form $\left(e \tau_{\bullet}(1.5-2.5) / n_{0} L_{n}^{2}\right) \nabla_{n}$, and $\left(e r_{\bullet}(1.5-2.5) / n_{0} L_{n} L_{T_{e}}\right) \nabla_{n}$ in addition to a pinch tersa on the order of the neoclassical value. The strong scale-length dependence can be

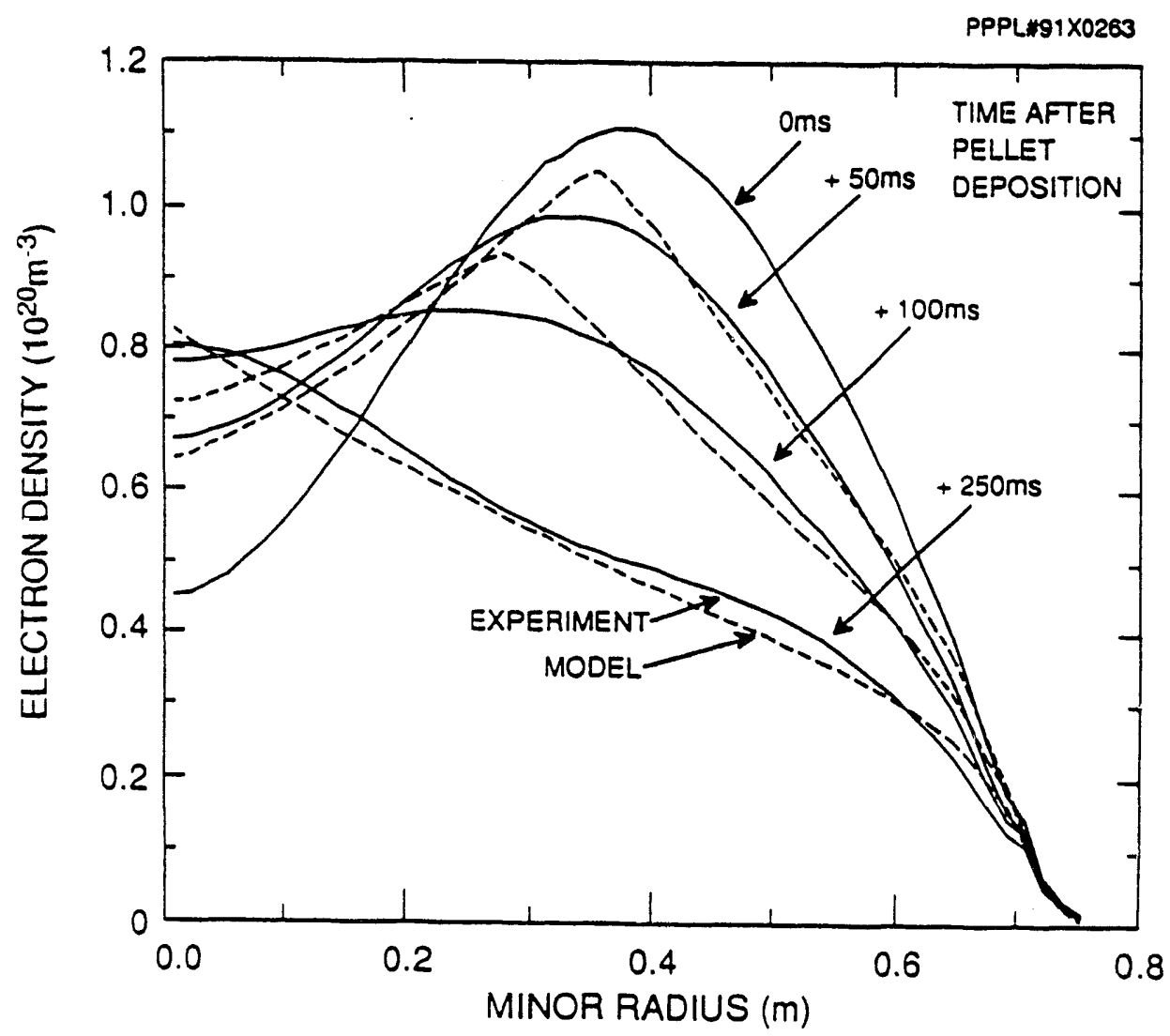

Fig. 7 The evolution of the measured density proflle after pellet injection compared with modelling assuming $D$ $2.4 \times 10^{19}(E / R)\left(T_{\theta}^{2} / n_{\theta} L_{n}{ }^{2}\right)$ and the neoclassical pinch. 
reduced if the local $q$ is included in the expressions. These expressions are consistent with electrostatic turbulent transport models (Ross et al., 1987).

Whether the highly non:inear dependence of the particle flux on density scale length and other terms indicated by the gas puff perturbation experiments is consistent with the density profile response to much larger amplitude perturbations has been studied using the relaxation of density profiles following deuterium pellet injection (Hulse et al., 1991). A beam-heated plasma fueled by six pellets presented a wide range of donsity scale lengths, including evolution from strongly hollow to strongly peaked profiles dith a central density up to - 10 times that in the gas puff experitients. As shown in Fig. 7, the evolution of the density profile sollowing one of the pellets is reasnnably well predicted using a transport model with the same functional form as that derived from the gas puff analysis. While the present level of agreement does not provide proof that this particular erangport model is uniquely correct, it does provide encouragement that highly nonlinear models consistent with theoretical expectations for electrostatic turbulent iransport $=a n$ be used to interpret and model density transport over a substantial range of plasma conditions. However, in the core of supershot discharges, this particular model overestimates the observed fluxes.

A related tzansport issue of considerable importance to ITER is the transport of trace impurities, in particular helium and iron. Charge-exchange recombination spectroscopy (Synakowski et al., 1990; Stratton, et al., 1991) has bion used to measure the evolution of the hellum and helium-like iron profiles after either the injection of a short gas puff or the injection of iron using the laser-blow-off sechnique. There are important differences between the behavior of the electron

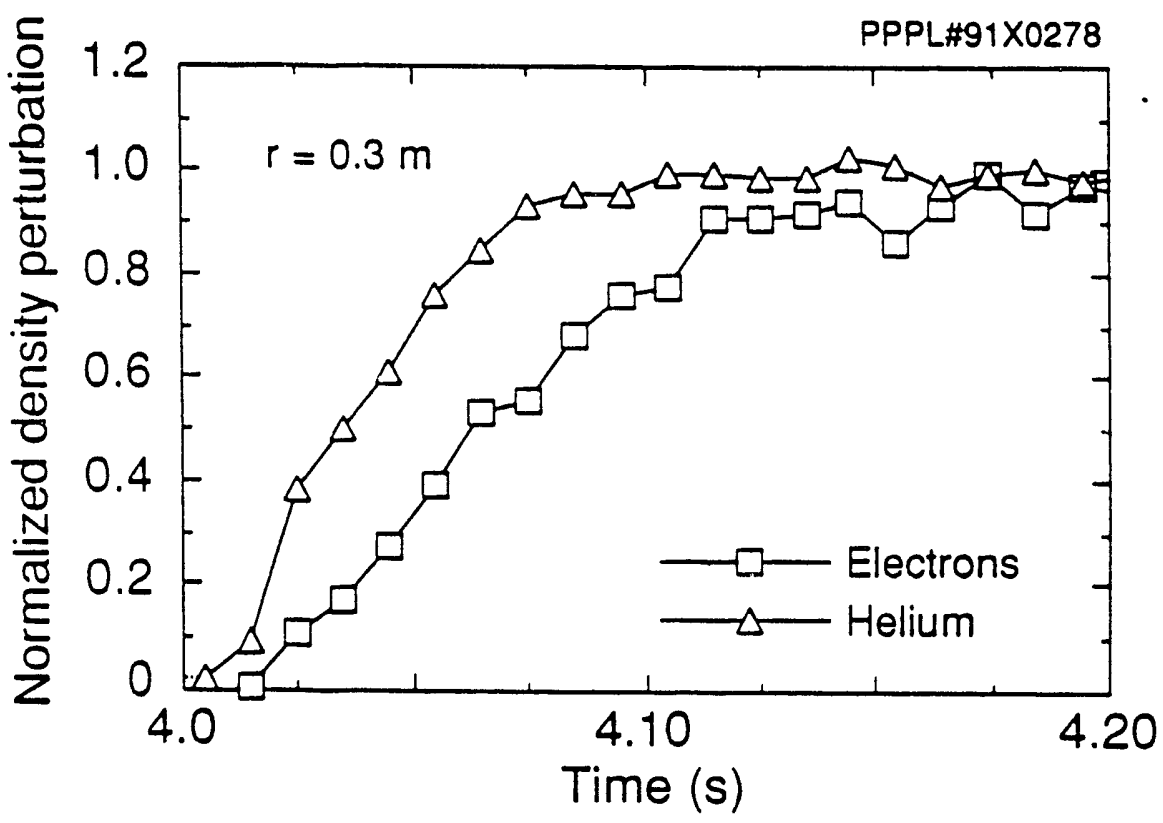

Fig. 8 Evolution of the perturbed electron density and helium density normalized to theit final levels at r/a $=0.38$ following a short He gas puft into a series of similar L-mode plasmas ( $\left.R_{p}=2.45 \mathrm{~m}, I_{p}=1.0 \mathrm{MA}, R_{\mathrm{NBI}}=13 \mathrm{MW}\right)$. 
perturbation and impurities. As shown in Fig. 8, the helium density and the perturted electron density are found to have a different cemporal behavior suggesting that the background ions (deuterium and carbon) are responding to achieve shazge neutrality. In addition, as shown in Fig. 9, the helium profile in the supershot jischarge is found to be peaked despite the fact that the fueling is from she edge for both the L-mode and the supershot discharges. The intrinsic carbon impurity profile in the supershot discharges is also peaked. This data suggests that the He flux should be described by both a dfffusive and convective flux since the source term on axis is negligible Assuming that $\Gamma_{\mathrm{He}}-\mathrm{D}_{\mathrm{He}} \nabla_{\mathrm{H}_{\mathrm{H}}}+\nabla_{\mathrm{He}} \boldsymbol{n}_{\mathrm{He}}$ then both $D_{H e}$ and $\nabla_{H e}$ increase with minor radius. The resulting value of $\nabla_{H}$ is significanty larger than the predicted neoclassical value and $D_{H e ~}-\chi_{l}{ }_{1}$. Similar resultg have been obtained in iron impurity injection experiments. The difference in tzansport between the electrons and impurities is qualitatively consistent with detailed computations of toroidal drift microinstabilities of the type described by Rewoldt and Tang (1990). In this quasi-linear analysis, electrostatic fluctuations are primarily driven by the spatial gradients of the electrons and ions. These Eluctuations in turn account for the transport of the impurity ions. Initial

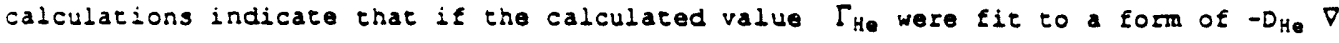
$\mathrm{n}_{\mathrm{H}}+\mathrm{v}_{\mathrm{He}} \mathrm{n}_{\mathrm{He}}$, then a significant value of the inward pinch would result.

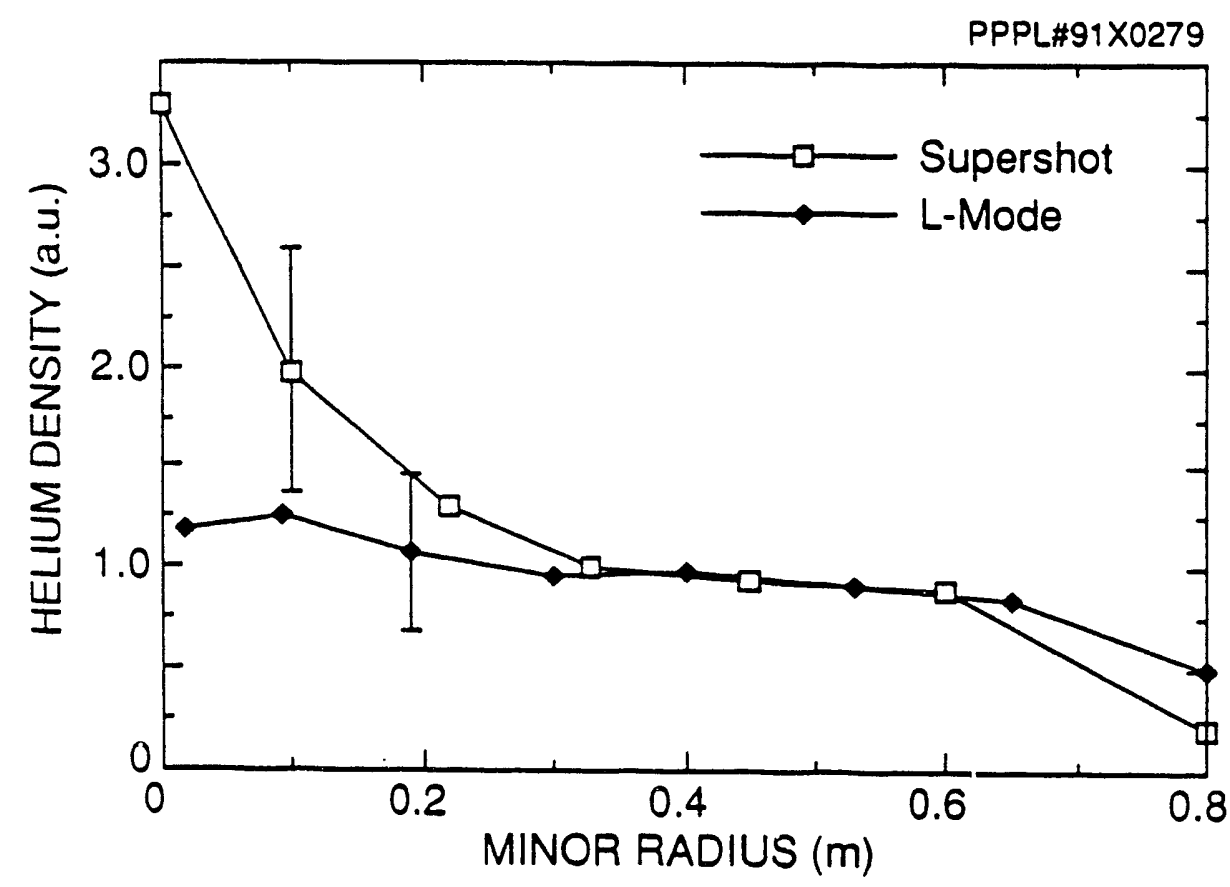

Fig. 9 Comparison of quasistationary He profilee in L-mode and supershot discharges $150 \mathrm{~ms}$ after a shurt He puff. The profiles are normalized to each othe, at $=0.4 \mathrm{~m}$. For both discharges $R_{P}=2.45 \mathrm{~m}, I_{P}=1.0 \mathrm{MA}, P_{\mathrm{NBI}}=13 \mathrm{MW}$.

\footnotetext{
The previous discusgion suggests that the simple model of transport driven by Electrostatic drift instabilities could account for the experimentally observed zrends. However, the use of such simple analysis estimates cannot be universally justified because the observed experimental values can vary over a large range in collisionality, density scale lengeh, and $\beta$ among other paramoters of importance. In supershot discharges, $\chi_{i}^{e t}(\tau=$ a/3) is found to decrease with lncreasing
} 
temperature as the density scale length deczeases from that in L-mode discharges. rhis ig in marked contrast with the results found in L-mode discharges: here both $z^{e::}$ and $x^{e::}$ increase with increasing temperature. These trends are shown in Eig. :0. For comparison, the upper bound on the transport of fast ions is shown. Using zee same xinetic stability code described earlier, rang and Rewoldt (19g1) have zalcuiated the cemperature variation of the diffusivity in L-mode discharges with ozoad density profiles and in supershor discharges with peaked density profiles. These initial cesults are encouraging in that they indicate the expected unfavorable :on semperature dependence in $L$-mode but a weak (though not favorable) dependence in supershot discharges. The well known difflculties with electrostatic models are the yariation with minor radius and the scalings with density, toroidal field, plasma zurrent and ion mass. While experimentally the thermal diffusivity increases signifisantly with minot radius, the electrostatic models predict that it should tecrease with radius, particularly over the outer half of the plasma. Recent density scans in $\mathrm{ICH}$ and beam-heated L-mode discharges at fixed power and current aze not consistent with a simple analytic relationship of the form $\chi^{05}-T_{0} i .5-$ $2.5) /\left(L_{n}{ }^{2} n_{0}\right)$ or $T_{0}(1.5-2.5) /\left(L_{n} L_{\tau_{0}} n_{0}\right)$ where we define $x^{e f t}=-\left(q_{1}+q_{0}\right) /\left(n_{1} \nabla r_{1}+n_{0} \nabla\right.$ $\left.\bar{F}_{0}\right)$. The thermal stored energy at fixed power scales weakly with $\bar{n}_{0}$ which is inconsistent with $x^{\text {ett }} \times \mathrm{T}_{0}{ }^{2}$ since $\mathrm{T}_{\bullet}(0)$ is nearly inversely proportional to the density. Detailed analysis of these density scans will provide another test of the xinetic stability code in the near future.

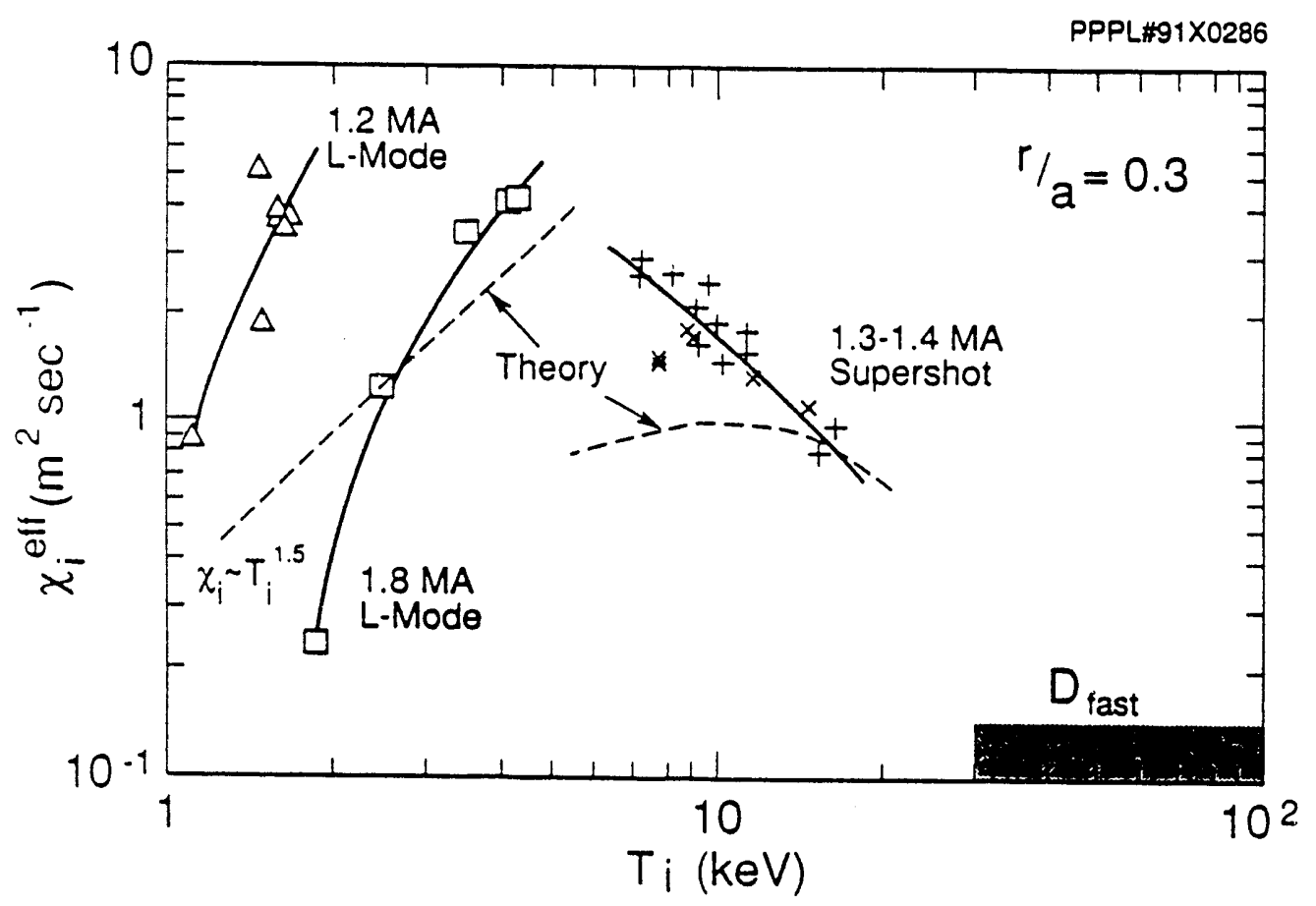

Fig. $10 x_{1}^{\text {plt }}$ versus ion temperature for $L$-mode and supershot discharges compared with the theoretical predictions of Tang and Rewolde (1991). The upper bound sor the diffusivity of fast ions, discussed in jec. III, is also shown. 
The observation of enhanced confinement in experiments with peaked density profiles (peliet-fueled, IOC, counter neutral beam injection on ASDEx, supershots, and the ZET $P E P$ mode) and the improvement of confinement in the ion channel has focused atsention on the cole of lon temperature gradient driven turbulence (ITGDT). (See stambaugh et al., 1990 for a review of work in this area.) This was reinforced by the work of Zarnstorff et al. (1989b) who observed that in TETR supershots in steady-stace $\eta_{1}-\eta_{1} c$ as predicted by Romanelli (1989) and Hahm and Tang (1989), although the predicted radial profile and magnitude of $\chi_{1}$ for ITGDT were found to be in disagreement with experimental measurement in L-mode discharges. This motivated a series of experiments (zarngtorff ot al. 1990b, 1990c; Efthimion et al. 1991) in which a supershot target, where iniclally $\eta_{l}-\eta_{1} c$, was perturbed by the injection of either carbon or deuterium pellets or by a short burst of gas such that $\eta_{1}$ became zansiently much larger than $\eta_{1}{ }^{c}$. As a result of the perturbation, Ine increased by a factor of -8 and the $I_{1}$ profilo becamo slightly steeper ( $L_{T 1}$ decteased). What was expected was a dramatic increase in the value of $x_{1}$, that would force the profiles back to "marginal stability" $\left(\eta_{1}-\eta_{1}^{c}\right)$, since the predicted value for the lon heat transport was much larger than observed. The analysls by zarnstorfe ot al. (1990b) using TRANSP indlcates that $\chi_{1}^{\text {tt }}$ was net changed by the perturbation. Analytic suid-type calculations of $x_{1}$ for the fully turbulent ITGDT eransport (Biglari et al., 1990) are a factor of 3 to 30 greater than observed implying that marginal stability should be enforced. However, kinetic quasi-linear numerical calculations (Rewoldt and Tang, 1990) in toroidal geometry of drift-modes driven by both ion temperature cradients and electron dynamics indicate that the total transport associated wi:h the most unstable modes should be approximately unchanged by the perturbation aid that the transpirt is much lower than the fluid predictions. These zesults suggest that nonlinear fluid theories of ITGDT transport might be brought into agreement with the experiment by a better treatment of toroidal and kinetic effects. In any case, further work is required to develop a reliable predictive capability for the observed transport.

Empirical global scaling relationships flt a broad range of experimental (L-mode) conditions from varlous machines reasonably well. However, a similar unifying zelationship for the local diffusivity has not been achieved. As dlscussed above, this may be a consequence of the transport lluxes being highly nonlinear or perhaps being enforced by a marginal stability requirement. Another possibility is that the ransport is non-local, meaning that the local transport coefficients are determined not by local quantities (such as $v^{*}$, B. $L_{n}$ or or $L_{T l}$ etc.) but by the value of such quantities elsewhere within the discharge. One example of non-local phenomena is the profile redistribution associated with the sawtooth instability. The possibility that non-local transport may play a zole in the quiescent region of a discharge has been ralsed by an old result and has been brought to attention by several recent experiments as well. Previously, Fredrickson et al. (1987) and Taylor et al. (1989) reported that the electron temperature scale length is a weak function of heating proflies and, outside the $q-1$ surface is a weak function of $q$. This concept of "proftle consistency" lor racher "profile resiliency", which recognizes the fact that the proflles are not rigidly constant) provides a good description of the electron temperature profile. In L-mode discharges, Scote et al. (1990c) observed that $x^{\prime t}$ and $x_{1} t^{t}$ decreased everywhere when the plasma current was increased. This is surprising because the calculated current density in the core toes not shange appreciably as the plasma current is increased due to the sawteeth phenomena. One might, therefore, have expected the change to be predominantly in the outer half of the plasma where the change in the current density, or q profile, is greatest.

The above results, cogether with the thooretical problem that electrostatic drift wave models do not predict the observed curzent scaling, motivated an experiment by 
zarnstorff et al. (1990b) to perturb the current profiles locally and to evaluate now the local transport evolved. Plasmas in approximate steady-state were contrasted with cases where Ip was rapidly changed from 2MA to $1 M$ or vice-versa during 2 seconds of balanced neutral-beam heating, as shown in Fig. 11. During the steady state and $I_{p}$ ramp-down conditions, the line-average density, $\bar{n}_{0}$, was maintained at a constant value by gas puffing. The rapid change in Ip produces large transient variations of the inferred fu and $q$ profiles as demonstrated by the change in the calculated internal inductance $l_{1}$. The energy confinement times, $\tau_{E}$, of the constant-current plasmas are in good agreement with l-mode scallng. Sollowing the cufrent campdown or rampup, the energy confinement time does not sollow the L-mode relationship $\left(\tau_{\varepsilon} \frac{L}{\varepsilon} I_{p}\right)$, but relaxes towards it only on a time-scale comparable to the resistive equilibration time, which is several seconds for the case shown, much longer than the energy confinement time itself. Since Bp and $q$ hear the edge of the plasma depend on $I_{p}$ and not (to lowest order) on the current profile, this indicates that $I_{p}$ scaling is not due to a direct dependence on the edge value of these quantities.
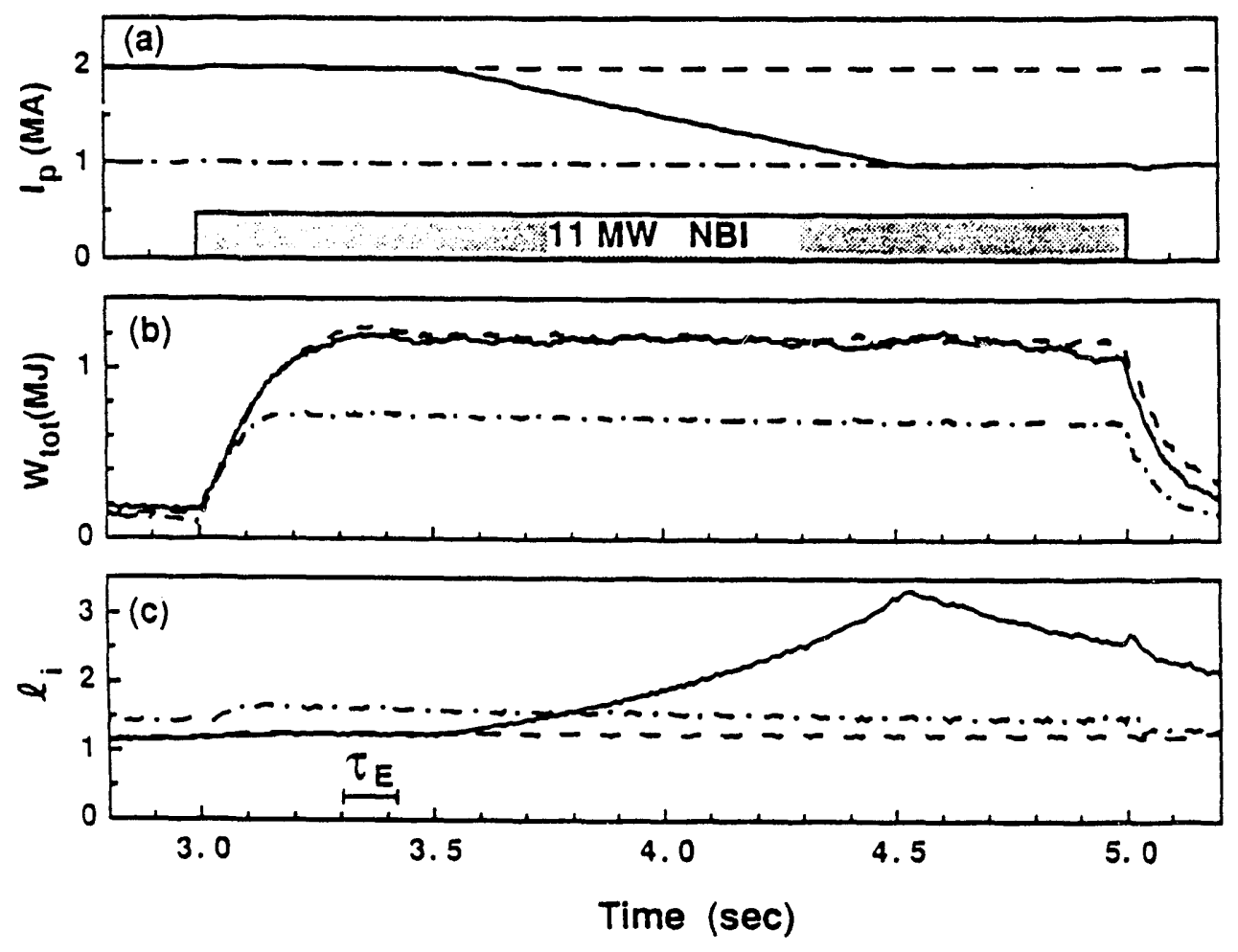

Eig. 11 Time evolution of (a) $I_{p}$, (b) $W_{t o t}$ measured by the diamagnetic loop, (c) il from TRANSP modelling for deuterium plasmas with $I_{p}-1 \mathrm{MA}, I_{p}=2 \mathrm{MA}$ and $I_{p}$ decreased from 2 to 1 MA. For these plasmas $B_{t}=4.8 T$, $R=2.45 \mathrm{~m}, \mathrm{a}=0.8 \mathrm{~m}$ and $\bar{n}_{0}-3.4 \times 10^{29} \mathrm{~m}^{-3}$.

The evolution of the current profile in these plasmas has been calculated by TRANSP The calculations indicate that a skin current penetrates to a/2 lfor the plasma shownl during the avallable beam-heating pulse. This is confirmed by the observed 
evolution of the sawtooth inversion radius, indicative of the $q$ - 1 radius, which is just beginning to change at the end of the beam-heating pulse. The changes in the $\mathrm{f}$ profile are calculated to transiently increase (decrease) the shear $\hat{s}$ ( $(r / q) \partial q / \partial r$ py a factor of -2 during the $I_{p}$ decrease (increase) sequence. During the change in $i_{p}$ and the resistive penetration of the current perturbation, the measured $I_{\text {e, }} I_{\text {! }}$ and $n_{e}$ profiles are not affected until the current perturbation reaches the plasma

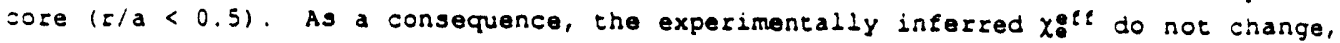
implying that the transport in the outer region $(r>a / 2)$ cannot depend strongly on the local jil, $q$, or $\hat{3}$. In particular, these observations imply that, as presently understood, transport due to resistive ballooning modes (Carreras and Diamond, 1989 ) (which varies as $-q^{8 / 3 /} \hat{s}$ ), neoclassical pressure-gradient turbulence (Kwon, Diamond, and Biglari 1990 ) (which varies as $\sim q^{7 / 3 / \hat{s}}$ ), or the Rebut-lallia model (Rebut et al., 1989) (which varies as ( $8 \mathrm{r}_{\text {e }}$ - C) $q / \hat{s}$ with $C$ varying as jul/2/q), cannot be significant in these plasmas in this region. These observations indicate that Lmode $I_{p}$ scaling is due to the curzent proflle in the core of the plasmapperhaps near the $q-1$ surface. Thus, the observed steady-state dependence of $\chi_{0}$ on $I_{p}$ across the entire profile must be due either -0 an additional parameter, correlated with $I_{p}$ in steady state, or a non-local cransport dependence on the current profile, pernaps due to a global instability or ladial turbulence propagation. In addition, they indicate that confinement can be substantially enhanced (or degraded) relative $=0$ - -mode scaling for a fixed $I_{p}$ value ty modifying the current profile.

\section{NONDIMENSIONAL SCALING EXPERIMENTS}

scaling studies cast in the form of nondimensional parameters not only offer the promise of providing a more physics-based approach to extrapolating to a future device, but may provide insight into the nature of the turbulence responsible for the transport. General considerations developed by Kadomtsev (2975) imply that global confinement should vary according co: $\Omega_{1} \tau_{E}=E\left(p^{*}, v^{*}, \beta, z_{0}, q_{1} \ldots \ldots\right)$ where $\Omega_{i}$ is the ion gyzo erequency and $F$ is a function of averages over the profile of "nondimensional" quantitlos. Perkins (1990) has ldentified lg nondimensional parameters which could potentially influence transport. On TETR, scans in which all but one of these nondimensional quantities were held constant were performed (Waltz et a1. 2990al. Scans in the nomalized gyroradius, $\rho^{*}-p_{3} / a=\left(2 \mathrm{~T}_{0}(0) \mathrm{m}_{1}\right) 1 / 2 / \mathrm{eBa}$ for reactor typical values of $q, \beta$, and $v^{*}$ - but not $p^{*}$ itself - have been performed and are of particular interest for extrapolating to future devices. Theories which involve fine-scale curbulence with respect to $p^{*}$ would be expected to follow gyroreduced Bohm scaling: $\chi_{q B} \propto\left(p_{3}^{2} \Omega_{1}\right)\left(p_{g} / L\right) F_{q B}$ where $F_{q B} i s$ a tunction of dimensionless parameters and $t$ is a local scale length. Transport due to long wavelength : urbulence with respect to the device size would be Bohm-1ike with: $\chi_{B} \propto$ $\left.\mid \rho_{5}^{2} \Omega_{1}\right) \Sigma_{3}$.

comparing confinement in discharges which have $q, \beta$ and $v$ the same while varying the normalized gyroradius, $\rho^{*}$, requires that the following conditions be met in a $B$ ? scan at fixed plasma size: $n \times B^{4 / 3}$ and $T \times B^{2 / 3}$. Then $p^{*}$ varies as $B^{-2 / 3}$. In such a scan, Fyro-reduced Bohm scaling predicts $\tau_{E} \propto$ B whereas Bohm scaling predices $\tau_{E} \alpha$ $3^{i / 3}$. The experimental challenge in these experiments is to obtain the plasma conditions which maintain ald other relevant dimensionless parameters constant.

Two different $\rho^{*}$ scans have been performed on TETR, at a low and a high value of the normalized density $n_{0} / B_{T}^{4 / 3}$. In addition, scan in $v^{*}$ have been performed. In the $p$. scans, as $B_{T}$ and $n_{0}$ were increased, the power necessary to maintain the temperature at $T \propto B_{\mathrm{T}}^{2 / 3}$ scaled approximately as $\mathrm{BT}^{2}$. Within each scan, the global confinement was unchanged, consistent with L-mode scaling and closer to Bohm than gyro-Bohm predictions. Experiments on DIII-D have shown simliar resules for global confinement but the local diffusivity in those experiments showed a BT scaling 
zlosez to gyro-Bohm (Waltz et al., 1990b). The most recent DIII-D experiments indicace that the local thermal diffusivity could be represented by either Bohm and Jyro-Bohm scaling (DeBoo et al. 1991). In the TETR $\rho$ * scans, values of $x^{\text {ef: }}$ show no significant scaling with $B_{T}$, consistent with the weak scaling predicted by Bohm but inconsiscent with gyro-Eohm scaling.

Another way to look at sine scaling of local transport, which does not involve taking cadial derivatives of experimental data, is to consider the power flow qexp(r) = $Q_{1}(r)$ + $Q_{e}(r)$ through a magnetic surface normalized to what one would expect for gyro-Bohm and Bohm scaling when temperature profile shapes do not vary: $q_{\text {exp/qgB }}=$ $\left[\left(a^{2}-r^{2}\right) / r^{3} R\right] \times e^{2} B^{2} q_{e x p} / 32 \sqrt{2} \pi^{2} n r_{\bullet} 5 / 2 M^{2 / 2}$ and $q_{0 \times p} / q_{B}-p^{*}\left(q_{e \times p} / q_{g}\right)$. Here the radial Eactor in brackets is introduced for convenience to avoid a large range of values in Eig. :2, which shows the normalized power flows of the two predictions for the low and high density $p$ * scan and the $v$ * scan. The Bohm-like scaling better describes the observed dependences. The $v$ * scan shows the power flow to be independent of collisionality, at least when $v^{*}<1$. Analysis of the uncertainty in the evaluation of $q_{e x p} / q_{B o n m}$ indicates that most of the -catter at $r=0.5 \mathrm{~m}$ could be attributed to the uncertainty in the diagnostic measuremants and it is approximately constant with minor radius. In the $\rho^{\circ}$ scans, the electron and ion temperature proftle shapes were sairly constant; however, in the $v^{*}$ scan, though the electron temperature profile shape remained constant, the ion temperature profile shape did vary systematically.

For these 1 -mode discharges the power flow trrough the ion channel dominates. This suggests that the electron temperature shou:d be replaced by the ion temperature in the expressions for normalized power flow. When this is done, the Bohm normalization again describes the data better, although there is a somewhat larger scatter in the data. These results suggest that long wavelength turbulence is primarily responsible for crosg-fleld eransport in these L-more plasmas.

\section{TURBULENCE STUDIES}

The inference that the transport is due to electrostatic instabilities has motivated the development of diagnostics to study density perturbations. shree principal diagnostics, microwave scattering, beam emission spectroscopy, and microwave zeflectometry have been used. The extraordinary mode microwave scattering system (Bretz et al., 1988) is the most established of these techniques. Scattering in all regimes in she plasma interior shows that the fluctuation spectrum is dominated by values of $k_{\perp}<4 \mathrm{~cm}^{-1}$, which are not spatially well resolved by microwave scattering (Peebles et al., 1990). The measured $k$-spectrum falls approximately as $k_{1}{ }^{-3}$ in ohmic, L-mode and supershot discharges. In ohmic discharges the apparent width of the spectrum in frequency is generally somewhat greater than $\omega_{*}$ a $k_{\theta} T_{0} / \theta B_{t} L_{n}$ with a shift, $\omega_{3}$, always in the electron diamagnetic drift direction. In ohmic discharges, the largest shifts, $\omega_{3} / \omega_{\text {: }}$ up to 4 , have been observed in high density plasmas $\left(\bar{n}_{0}>3 \times 10^{19} \mathrm{~m}^{-3}\right)$. In beam-heated discharges, toroidal plasma rotation dominates the form of the frequency spectrum due to the instrumental averaging of the rotation profile over the scattering volume. The estimated magnitude of $\delta n_{e} / n_{e}$ - 0.3 at $\mathrm{c} / \mathrm{a}=0.3 \mathrm{ig}$ in approximate agreement with the mixing length estimate $L /\left\langle k_{\perp}>L\right.$ where $L=\sqrt{L_{n} L_{T}}$. This is consistent with theoretical estimates based on collisionless trapped electron mode turbulence. The scattered power at $5 / a=0.3$ for a Eixed value of $k_{\perp}-4.2 \mathrm{~cm}^{-1}\left(k_{\perp} p_{3}=0.5\right)$ has been found to increase with reutral beam heating power in $L$-mode discharges.

The beam emission spectroscopy diagnostic (BES) diagnostic measures plasma density Eluctuations by observing the fluorescence emitted from a noutral beam as it impacts the plasma (Paul and Fonck, 1990). The observed light from the Da transition 

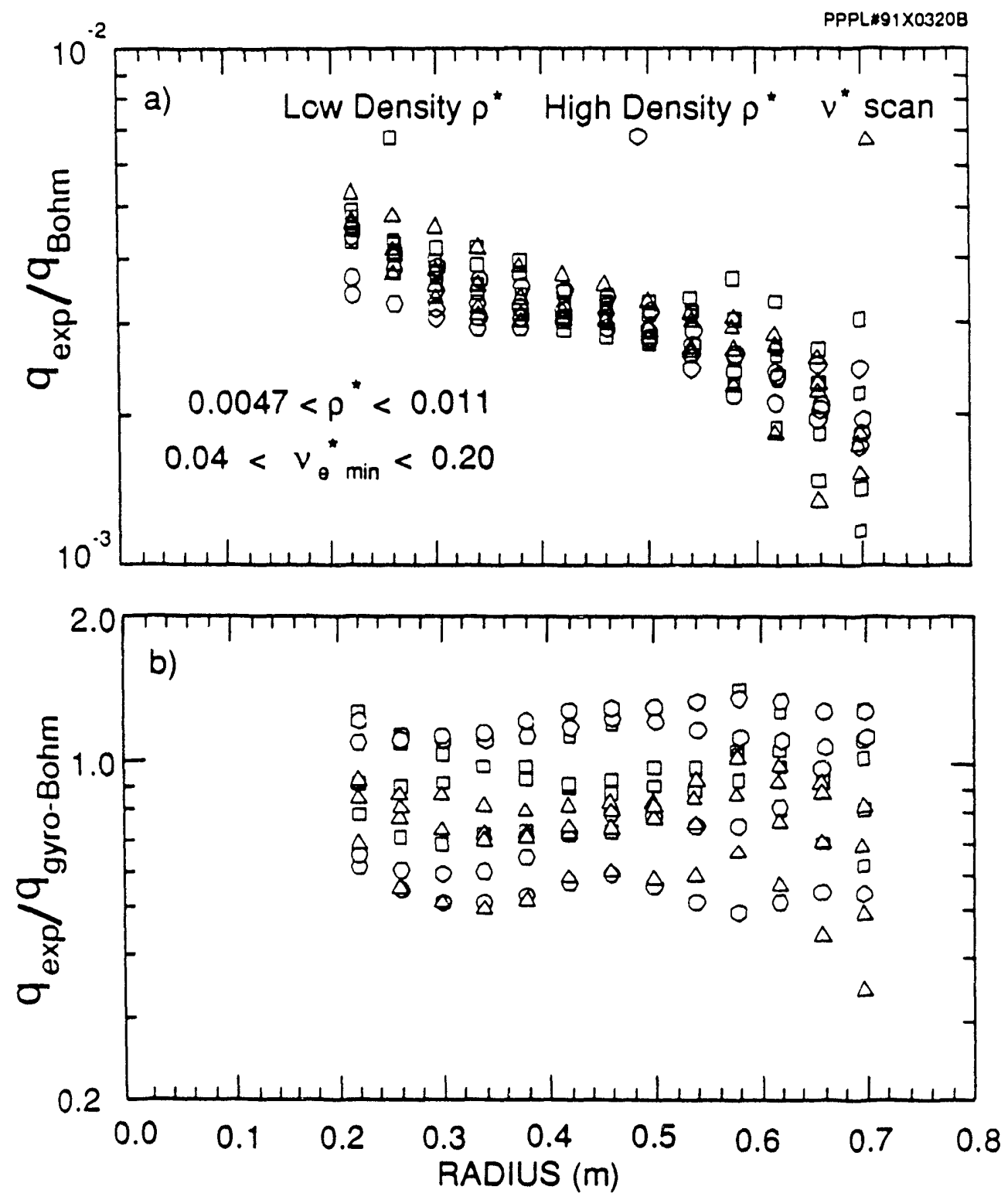

Flg. 12 The radial variation of the total heat flux normalized to the prediction of a) Bohm scaling and b) gyroreduced Bohm scaling for L-mode plasmas in the high and Low density $p^{*}$ scans and the $v^{*}$ scan.

$(n-3 \rightarrow 2)$, a cesule of collisioral excitation with the plasma ions, is proportional to the eluctuating density. This diagnostic enables the moasurement of isng wavelength turbulence $\left(k_{\perp}<2 \mathrm{~cm}^{-1}\right)$ with good gpatial resolution $(<2 \mathrm{~cm})$. Recent zesults d fo presented at this meeting by Fonck et al. (1991) on L-mode discharges. in the edge region, the densicy perturbations are large $(\tilde{n} / n-10$ ) and decrease to $\leq$ for $0.5<E / a<0.9$. The radial and poloidal correlation lengrhs decrease wish minor radius from values of about $10 \mathrm{~cm}$ at $\mathrm{r} / \mathrm{a}=0.5 \mathrm{co} 2 \mathrm{~cm}$ at $\mathrm{e} / \mathrm{a}=1$ 
Eurtnermore, as shown in Fig. 13, durfing a power scan in L-mode discharges, the densisy fluctuation amplitude at $r / a=0.7$ is found to be inversely correlated with the energy confinement time whereas no variation in $\delta_{n} / n$ with $\tau_{\mathrm{g}}$ is observed at the edge $(5 / a>0.95)$. This data indicates the existence of large-scale fluctuations correlated with enhanced transport. Such fluctuations are poorly resolved spatially by microwave scattering rechniques. Trapped-ion modes (Dlamond and Blglari, 1990; $3 i g l a r i$ and Diamond 1991 , and references thereln) are theoretically predicted to have these characteristics. Further microinstability calculations, which take into account long radial wavelength properties in toroldal geometry (Marchand et al., 19801 , will be performed.
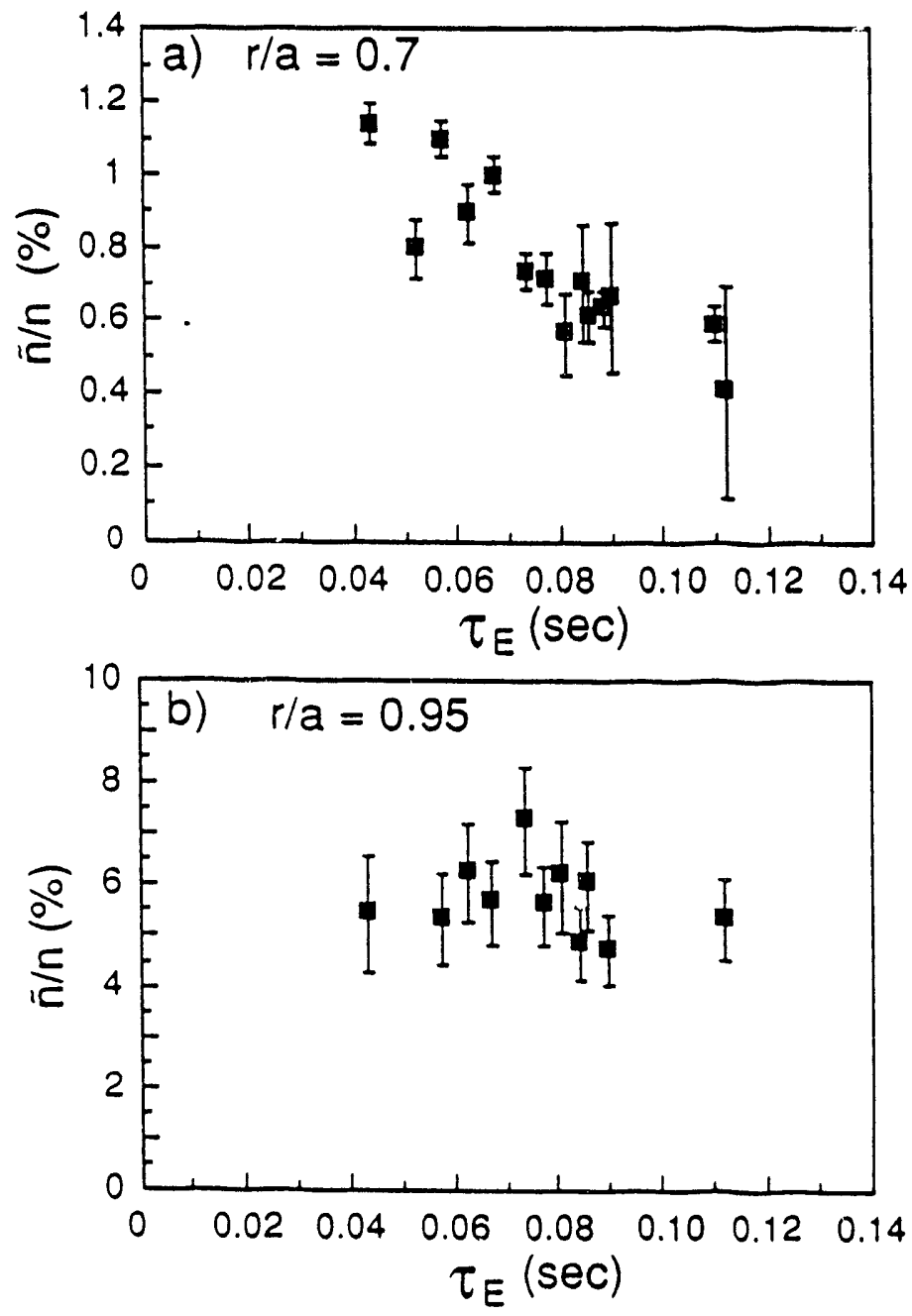

E:g. 13 Amplitude of local density fluctuations in L-mode discharges versus total confinement time cor a) an interior region of the plasma and b) the plasma edge.

:o extend Eluctuation studies to longer wavelengths, a single channel extraordinaryrode $140 \mathrm{GHz}$ reflectometer was installed at the end of the last operating period and -s now being upgraded to four channels. Initial resules in ohmic discharges indicate that fluctuation levels in the plasma core for $x_{\perp}<1 \mathrm{~cm}^{-1}$ are very small, 
$\pi / n<10^{-4}$, and increase monotonically with minor radius to values of approximately $: 0^{-2}$ at the edge of the plasma. Edge density fluctuations with $k_{\perp}-1 \mathrm{~cm}^{-1}$ and $\tilde{n} / \mathrm{n}$ up to 108 have also been observed near the inner wall using visible imaging of ambient $H_{\alpha}$ light (2weben and Medley, 1989).

These initial zesults indicate that in ohmic discharges a peak in the fluctuation spectrum may exist between $1<k_{1}<4 \mathrm{~cm}^{-1}$. Experiments with the full complement of zeflectometry channels will be performed to complete the study of the k-spectrum and perform a comparison of the results of the different diagnostics in different operating regimes.

VIII. ROLE OE MHD IN TRANSPORT

MHD activity plays an important role in determining plasma performance. For example, performance of supershot discharges is found to be correlated with the stabilization of the sawtooth instability and avoidance of low $\mathrm{m} / \mathrm{n}$ activity. vigcharges with the highest values of density profile peakedness havis the longest confinement times and are observed to be sawtooth stabilized. The beam power zequired to stabilize the sawtooth instabllity increases with decreasing q(a) (Manickam et al., 1989). The confinement of high current (>2MA) discharges has been, thus far, impaired by the onset of sawteeth. ICH stabilization of sawteeth, which was first demonstrated on JET (Campbell et al. 1988), shows great potential for extending the operating reglme to higher current (hosea et al., 1990a, 1990b). Recent experiments at modest powers (PNBI - IIMW and $P_{I C H}$ - 3MW) have demonstrated that it is possible to obtain supershot conditions with ICH and to stabillze the gawtooth instability at relatively high current (2Mi), as shown in Fig. 14 (Phillips et al., 19911 .

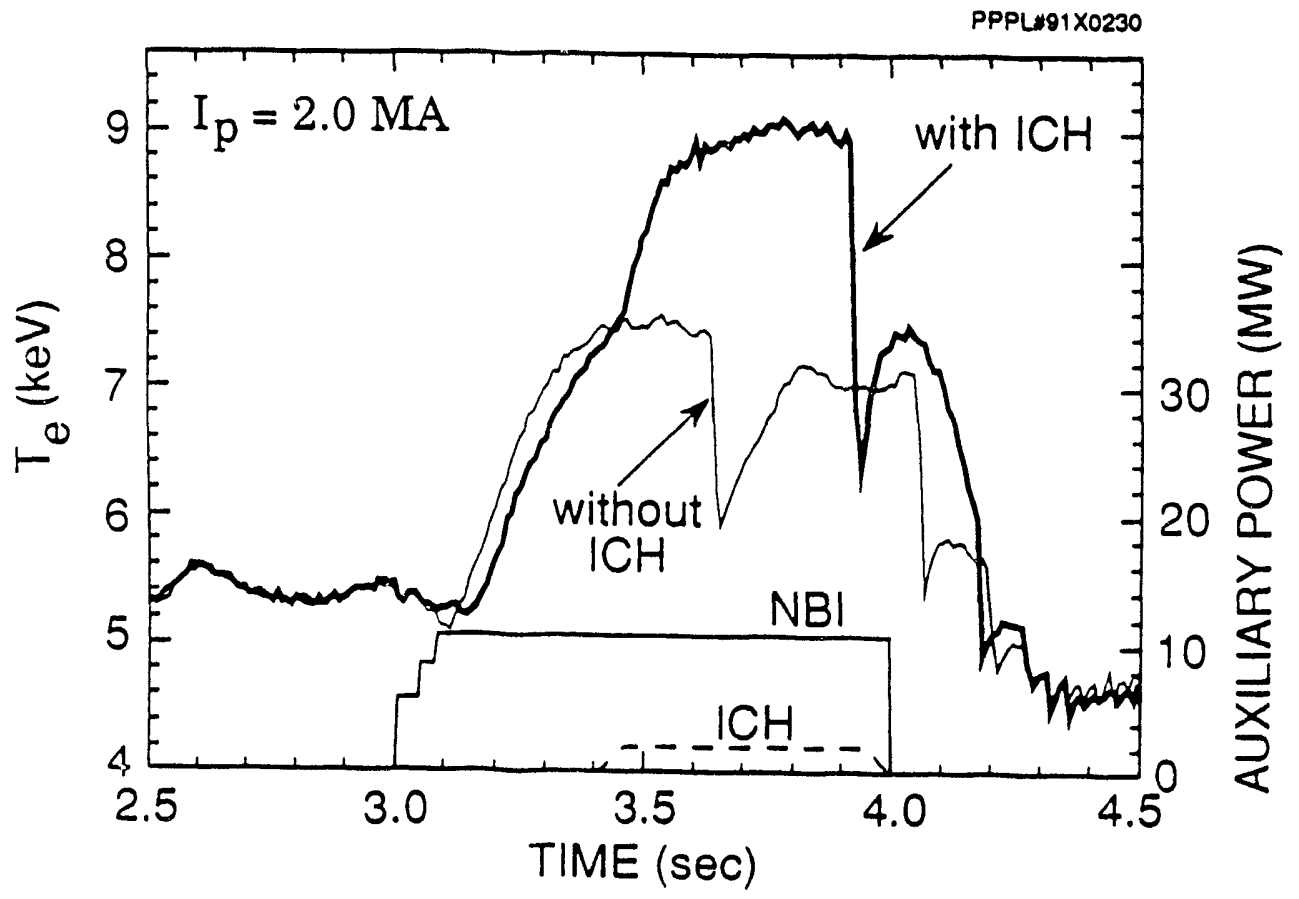

Eig. 14 sawtooth stabilization of a $2.0 \mathrm{ma}$ supershot plasma produced with liM of NBI and $3 \mathrm{MW}$ of $I C H$. $\left(R_{\mathrm{p}}-2.62 \mathrm{~m}\right.$, $\bar{n}_{0}-2.8 \times 1019 \mathrm{~m}^{-3}, \mathrm{D}\left({ }^{3} \mathrm{He}\right)$ regime). 
The propagation of heat after the crash phase of the sawtooth instability has been studied by Fredrickson et al. (1990) who have modified theif previous interpretation (Eredrickson et al., 1986) that the value of heat diffusivity, $\chi^{\mathrm{HP}}$, from an analysis If the heat pulse, is larger than that from the power balance, $\chi^{p}$. The recent analygis shows that the temperature increases beyond the mixing radius after the sawtooth crash. This iniclal ballistle response has been modeled by a large zansient increase in the heat diffusivity. The subsequent propagation of the heat pulse after this redistribution is found to be in reasonable accord with a model that assumes $\chi^{H P}-\chi^{P B}$. Whether the increase in the heat diffusivity accompanying ine sawtooth crash is due to magnetic stochasticity or due to an increased level of trift wave turbulence remains to be resolved. Nazikian et al. (1991) have observed short wavelength, high frequency $\left(-w_{*}\right)$ turbulence in the region of the $x$-point of zhe $m=1$ istind during the sawtooth crash.

Ear more deleterious than sawtooth activity is the onset of low-m modes which have set the operational boundaries of the sufershot regime $\beta_{n}=\left(\beta /\left(I_{p} / a B_{T}\right)<2.7\right.$ $(3 \mathrm{mT} / \mathrm{MA})$ and $\epsilon \beta_{p}<0.7$ (McGuire et al., 1987; Bell et al., 1989). These snscabilities can result in either a soft $\beta$-collapse (n 2 l) or (less frequently) a rapid disruption ( $n-1)$. In recent experiments (Navratil et al., 1990; Sabbagh et a1., 1990 and $199 i$ ) the current was ramped down just prior to neutral beam infection which significantly increased $l_{1}$ during the heating. This appeared to suppress the Eelecerious MHD activity and substantially extended the TETR limits in both $\beta_{n}$ and $\varepsilon \beta_{p}$ at lo.v plasma current. As shown in $51 \mathrm{~g}$. 15, $\varepsilon \beta_{p}$ was increased to 1.6 and $\beta_{n}$ to 4.7. In the discharges with large values $\ldots$ E $\beta_{p}$, a natural inboard poloidal

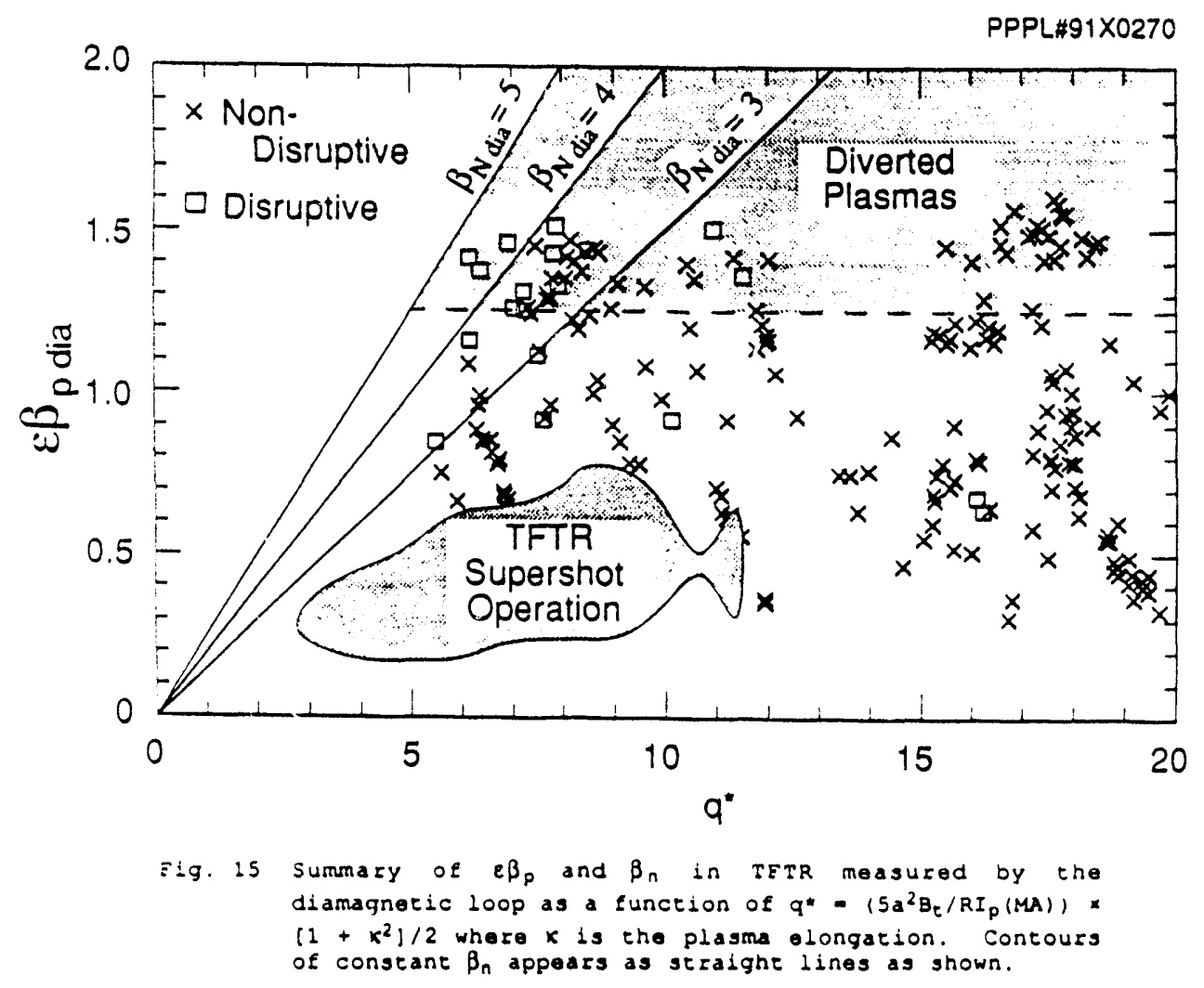


ilyertor appeared. These discharges have enhanced confinement with $\tau_{E} / \tau_{E}^{L} \leq 3$. This enhancement cends to increase as $\varepsilon \beta_{p}$ increases. Discharges in which the plasma zurzent is ramped down have the largest improvement over L-mode. This operating zegime demonstrates that peaking the current profile can both increage the entrgy zonfinement time and extend the tokamak operating regime.

in addition to affecting the confinement of the background plasma, MHD instabilities zan interact with the fast ions. Zweben ot al. (1990) have observed that the fusion products are expelled from the plasma when large amplitude low-m instabilities are present and during the crash of the sawtooth. This MHD activity does not appear to se driven by the fast particles. However, since collective interactions driven by East particles, such as the fishbone instability, have been observed frequently on smaller devices, there are concerns that during deuterium-tritium operation, alpha particles could collectively interact with MHD modes. An instability with a very Low predicted threshold (Cheng et al., 1990) is the toroidal Alfven eigenmode which zan be destabilized by the alpha particles with parallel velocity $V_{\alpha} \sim V_{A}$. Wong et a). (1991a; 1991b) have recently performed a simulation exferiment using neutral seams which is reported at this conference. At low toroidal field $(-1 \mathrm{~T})$ and (relatively) high density $\left(\bar{n}_{0}-2.5 \times 10^{19} \mathrm{~m}^{-3}\right.$ ) the theoretical condition for instability $V_{b}-V_{A}$ can be satisfied. Bursts of MHD activity in the frequency range of the coroidal Alfven eigenmode were observed when the plasma density was sufficiently high that $V_{b} / V_{A}>0.7$. The observed frequency scaled as the theoretical frequency when varied over a factor of two by varying the plasma density

PPPL \#90T0095

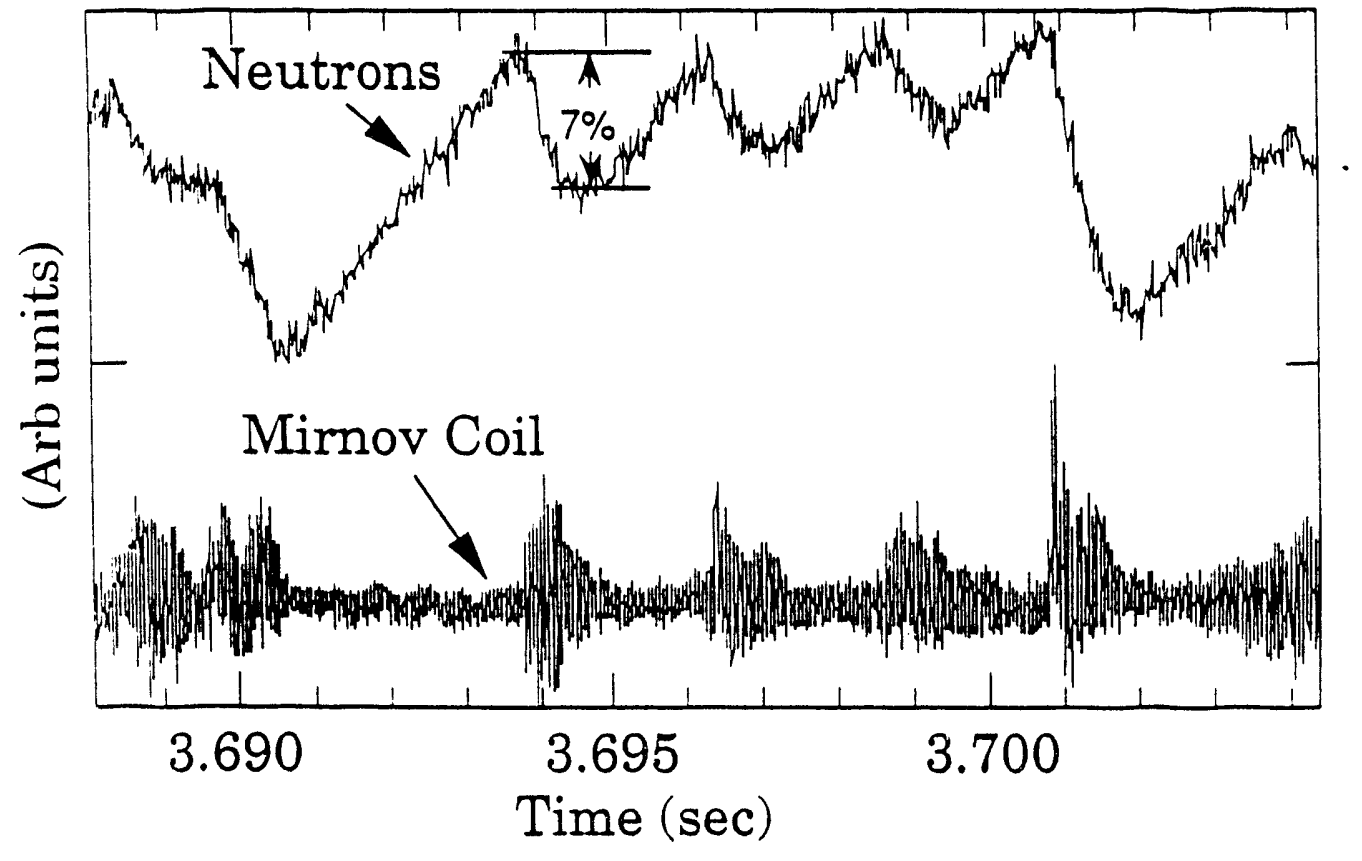

Fig. 16 Correlation between total neutron emission rate and Mirnov coll signal during injection of an energetic (lookeV) neutral beam into a relatively high-density $\left(\bar{n}_{0}-2 \times 1019 \mathrm{~m}^{-3}\right)$ plasma at low toroldal flold (BT 1T). The vertical scale for each trace is llnear with a suppressed zero. 
and the toroidal field. Beam emission spectroscopy was used to measure the mode structure of the instability inside the plasma (a/2 < 5 < a) which was found to be as expected for a global eigenmode. These experiments also showed a decrease in neutzon flux with the occurrence of the MHD fluctuation, indicating the loss of fast beam ions from he plasma, as shown in Fig. 16. Initial estimates indicate that the instability thrt isld is substantially higher than the theoretically predicted inreshold; however, further theoretical work is in frogress (Berk et al. 1991 ).

\section{WALL CONDITIONING}

Wall conditionsng techniques have been and continuf to be a key element in the development of the enhanced confinement regime in TFTR. Ohmic He conditioning shots (Dylia et al., 1987) continue to be used to maintain low recycling of the hydrogenic species. Once the hydrogen recycling has beer. reduced, plasma performance becomes well correlated with the level of CII impurity light from the target plasma. Previous problems with carbon blooms during high-power supershots have been remedied by replacing the graphite tiles in the high heat flux region of the toroidal limiter with carbon-carbon composite tiles which have much higher thermal stress capability (U:rickson et al. 1989, 1990). Further reductions in the influx of carbon were achieved during the 1990 run by injecting lithium pellets prior to beam injection. Snipes et al. (1991) at this conference describe the use of pellets to coat the walls with ilitium. The result of the lithium injection is to decrease tho secycling of carbon and deuterium leading to appreciable, and reliable, increases in the central ion temperature, total plasma stored energy, and peak neutron rate. Uaing this technique, ths maximum neutron source strength of $j \times 10^{16}$ neutrons per second, corresponding to a D-D fusion power of $58 \mathrm{~kW}$, was achieved with $33 \mathrm{MW}$ of heating power. In this discharge, the value of $Q_{D C}=1.8 \times 10^{-3}$ was among the highest achieved on TFTR. Transport analysis indicates that the improvement with li pellet injection is mainly due to a reduction in the ion thermal diffusivity by 30 oot in the off-axis region $0.25<\mathrm{r} / \mathrm{a}<0.5$. How a relatively minor change to the plasma bouncary condition results in a significant change to the core transport soefficients zemains a major unanswered question.

\section{SUMARY}

Transport studies in L-mode, supershots and H-mode suggest that in discharges without observable MHD activity, the underlying transport is caused by electrostatic surbulence. The relationship between $x_{\phi}^{e t}, x_{1}^{e f t}, x_{0}^{\text {elt }}$ and the particle diffusivities and the limits placed on the convection multiplier indicate that strong magnetic stochasicicity does not govern transport. The experimental results also indicace that existing theoretical models need to be further developed. The observation of long-scale-length fluctuacions being correlaced with confinement has significant physical ramifications. Further studies with additional diagnostics over a broader parameter range during the next run will be conaucted to explore these thoughtprovoking results further. The nondimensional scaling experiments indicate that Bohm-like scaling is a better description of the heat flux than gyro-reduced Bohm scaling. This study also tmplies that long wavelength modes may account fos the major part of the observed transport. The current perturbation experiments have shown that Ip scaling $i$. hot due to local changes in the q or $\hat{s}$ profile, implying that transport is non-local or that other parameters are subtly correlated and not sontrolied. Present resistive ballooning models as well as the Rebut-Lallia model do not describe these results. These experiments also indicate that confinement can De controlled through the central current profllo. Marginal stability imposed by fluid mudels of ITGDT has been tested by perturbation experiments which have temonstrated that a large increase in the ion heat diffusivity does not occur when the criteria for the onset of ITGDT are exceeded. These results indicate the need 
to include kinetic and toroidal effects in the analysis. Although the transport studies have not yet succeeded in developing a reliable theoretically-based predictive capabilily, they have significantly constrained the theoretical models.

The transport studies have stimulated the development of new operating regimes such as the high- $\beta_{p}$ regime, and have demonstrated the importance of current profile control in the next generation of machlnes. As always, there continues to bo an important role for exploration. The development of new wall coating techniques using lithium pellet injection has resulted in extension of the parameter range for neutron source strengeh and for $Q_{D D}$ in preparation for $D-T$ experiments on TFTR.

zreparations are actively underway to begin D-T operations in 1993 . Those experiments will be the natural consequence of the transport studies described here, for not only will they build on these experiments, but they will also enable tzansport to be investigated in a new regime. For example, the simulation experiments with neutral beam injection of the coroidal Alfven eigenmode showed that tho interaction of MHD anc sast ions can be an important process. When extrapolated to D-T, the experimental conditions achieved to date in TFTR will enable the direct study of collectively driven alpha instabilities (Budny et al., 1991). These instabilities could be important in the next generation of machines, BPX and ITER, and will be studied on TETR.

\section{Acknow Ledgements}

It is a pleasure to acknowledge the contribution of the many engineers and cechnicians whose efforts and personal sacrifices have enabled us to conduct the experiments described here, to J. D. Strachan for his many contributions, and to J. D. Callen and R. H. Diamond for many stimulating discussions.

\section{REEERENCES}

eli, M. G. et al. (2989). Plasma Phys. Control. Nucl. Fus. Res.. Proceedings of the Twelfth international Conference, Nice, 1988 (IAEA, Vienna) Vol. I, p. 27. serk, H. et al. (2991). At this conference.

Biglari, H., Diamond, P. H., and Rosenblueh, M. N. (1989). Phys. Fluids B $1,109$.

Biglari, H., and Diamond, P. H. (1991) To be published in Phys. Fluids B.

3iglari, H. and Chen L. (1991). Submiteed to Phys. Eluids.

3retz, N. et al. (2988). Rev. Sci. Instruments 52, 1538.

soivin, R. L. et al (1991). At this conference.

Boozer, A. H. et al. (1990). Phys. Fluids B 2, 2870 .

Budny, $R$, et al. (1991). At this conference.

Burrell, K. H. et al. (1990). Phys. Fluids B 2, 2904

3ush, C. E. et al. (1990). Phys. Rev. Lett. 65, 424.

callen, J. D. (1990). Rhys. Fluids B 2, 2869.

zampoell, D. J. et al. (1988). PHys. Rev. Lett. 60, 2148.

Cazreras, B.A. and Dlamond, P.H. (1989). Phys. Fluid B 2, 1011.

cheng, C. 2. (1990). Princetion Plasma Physics Laboratory Report PPPL-2717 (Oct.

19901 . To be published in Phys. Fluids $B$.

JeBoo, J. et al. (1991). At this conference.

Diamond, P. H., and Blglari, H. (1990). Phys. Rev. Lett. 65, 2865.

Dylia, H. F. et al. (1987). Nucl. Fusion 22, 1221.

EEtnimion, P. C. et al. (1989). Plasma Phys. Control. Nucl. Fus. Res.., Proceedings

of the Twelfth International Conf., Nice, 1988 (IAEA, Vienna) Vol. I, P. 307.

Etsnimion, P. C. et al. (1991a). Phys. Rev. Lett. 28, 421.

Efthimion, P. C. et al. (1991b). To be published in Phys. Fluids B.

Ezedrickson, E. D. et al. (1986). Nucl. Fusion 26, 849.

Eredrickson, E. D et al. (1987). Nucl. Fusion 27, 1897.

Ezedrickson, E. D. et al. (1990). Phys. Rev. Lett. 65, 2869

Eonck, R. J. et al. (1989). Phys. Rev. Lett. 63, 520. 
Fonck, R. J. et al. (1991). At this conference.

Soldston, R. J. et al. (1981). J. Comput. Phys. 43, 61.

soldston, R. J. et al. (1984). Plasma Phys. Control. Fusion 26, 87.

Goldston, R. J., white, R., and Boozer, A, (1981). Phys. Rev. Lett. $47,647$.

Grisham, L. R. et al. (1990). Controlled Eusion and Plasma Heating, Proceedings of

the Seventeenth European Conference, Amsterdam, 1990, Vol. 14B, Part I, P. 146.

jeisham, L. R. et al. (1991). Submitted for publication in Phys. Rev. Lett.

Groebner, R. J. et al. (1985). Nucl. Fusion 26, 543.

iahm, T. S, and Tang, W. M. (1989). Phys. Eluids B 1, 1185.

Hammett, G. (1986). Ph.D. Thesis, Princeton University (1986). Available from University Microfilm, Ann Arbor, MI 48106 USA.

Hawryluk, R. J. (1980). In Coppi, B. et al., editors, physics of plasmas Close to Thermonuclear Conditions, (CEC, Brussels, 1980 ) Vol. I, P. 19.

Hawryluk, R. J. et al. (1987). Plasma Phys. Control. Nucl. Fus. Res., Proceedings of the Eleventh International Conference, Kyoto, 1986 (IAEA, Vienna) Vol. I, P. 51. Heidbrink, W. et al (1991). In preparation.

Hieshman, S. P., and Sigmar, D. J. (1981). Nucl. Fusion 22, 1079.

Hosea, J. C. et al. (1990a). Proceedings of the Joint Varenna-Lausanne International Workshop on Theory of Fusion Plasmas, August 1990.

Hosea, J. C. et al. (1990b). Proceedings of the Thirteanth International Conference on Plasma Physics and Controlled Nuclear Fusion Research, Washington, 1990 (IAEA, Vienna) Paper IAEA-CN-53/E-:-5 to appear.

Houlberg, W. A. et al. (1990). Phys. Fluids B 2, 2913.

Hulse, R. et al. (1991). Presented at Transport Task Force workshop (Austin, IX).

Kadomesev, B. (1975). Sov. J. Plasma Phys. I, 295.

Kaye, S. M. et al. (1990). Phys. Eluids B 2, 2926

Kwon, O. J., Diamond, P. L., and Biglari, H. (1990). Rhys. Fluid B 2, 291.

Manickam, J. et al. (1989). Plasma Phys. and Control. Nucl. Fus. Res., Proceedings of the Twelfth International Conference, Nice, 1988, (IAEA, Vienna) Vol. I, $P$.
395 .

Marchand, R., Tang, W. M., and Rewoldt, G. (1980). Phys. Fluids 23, 1164.

Meade, D. M. et al. $(1990)$. Proceedings of the Thirteenth International Conference on Plasma Physics and Controlled Nuclear Eusion Research, Washington, 1990 (Vienna) Paper IAEA-CN-53/A-1-1, to appear.

McGuire, K. et al. (1987). Plasma Phys. Control. Nucl. Fus. Res., Proceedings of the Eleventh International Conference, Kyoto, 1986 (IAEA, Vienna) Vol. I, $P .421$.

Mynick, H. E., and Duvall, R. E. (1989). Phys. Fluids B $1,750$.

Navratil, G. A. et al. (1990). Proceedings of the Thirteenth International Conference on Plasma Physics and Controlled Nuclear Fusion Research, Washington, 1990 (IAEA, Vienna) Paper IAEA-CN-53/A-3-3, to appear.

Nazikian, R. et al. (1991). At this conference.

Eaul, S. E., and Eonck, R. J. (1990). Rev. Sci. Instrum. 61, 3496.

Peebles, W. A. et al. (1990). Proceedings of the Thirteenth International Conference on Plasma Physics and Controlled Nuclear Fusion Research, Washington, 1990 (IAEA, Vienna) Paper IAEA-CN-53/A-7-1, to appear.

Perkins, F. (1990). Princeton Plasma Physics Lab. Report PPPL-2708.

zhillips, C. K. et al. (1991). At this conference.

Radeztsky, R. H. et al. (1988). Controlled Fusion and Plasma Heating, Proceedings of the Eifteenth European Conference, Dubrounix, 1988. Vol. 12B, Part 1, p. 79.

Rebut, P. H., Lallia, P. P., and Watkins, M. L. (1989). Plasma Phys. and Control. Nucl. Fus. Res., Proceedings of the Twelfth international Conference, Nice, 1988 (IAEA, Vienna) Vol. II, P. 191.

Rewoldt, G. and Tang, W.M. (1990). Phys. Eluid B 2, 318.

Romanelli, F. (1989). Phys. Fluld B $2,1018$.

Ross, D. W. et al. (1987). IRSG Panel Report No. DOE/ET-53193-7.

Sabbagh, S. A. et al. (1990). Controlled Eusion and Plasma Heating, Proceedings of the Seventeenth European Conference, Amsterdam 1990, Vol. 14 B, Part 1, p. 387.

sabbagh, S. A. et al. (1991). To be published in Phys. Fluids B.

scott, S. D. et al. (1987). Controlled Fusion and Plasma Physics, Proceedings of the Eourteenth European Conference, Madidd, 1987, Vol 11D, Part 1, p. 65.

scott, S. D. et al (1989). Plasma Phys. Control. Nucl. Fus. Res.. Proceedings of the Twelfth International Conference, N1Ce, 1988 (IAEA, Vienna) Vol. I, P.655.

scott, S. et at (1990a). Phys. Rev. Lete. 64, 531.

Scott, S. D. et al. (1990b). Phys. Fluids B 2, 1300.

Scott, S. D. et al. (1990C). Proceedings of the Thirteenth International Conference on Plasma physics and Controlled Nuclear Fuston Rosearch, Washington, 1990 (IAEA, Vienna) Paper IAEA-CN-53/A-3-6, to appear.

Smithe, D. N., Colestock, P. L.,. Kashuba, R. J., and Kamach, T. (1987). Nucl. Fusion $27,1319$.

snipes, J.A. et al. ( 1 eqg1). At this conference. 
jtambaugh, R. D. et al. (1990). Phys. Eluids B 2, 2941.

stzachan, J. J. et al. (1987). Phys. Rev. Lete. 58, 1004.

Stzatzon, B. C. et al. (1991). Nucl. Eusion 31, 171.

synakowgki, E. J. et al. (1990). Phys. Rev. Lett. 65, 2255.

Takase, $Y$. et al. (1991). In preparation.

Tang, W. M. and Rewoldt, G. (1991). Presented at the Sherwood Meeting, Seattle, (April 1991 ).

Taylor, G. et al. (1989). Nucl. Fusion, 22, p. 3.

Jlfickson, M. et al. (1989). Plasma Phys. Concrol. Ndel. Fus, Res., Proceedings of the Twelfth International Conference, Nice, 1988 (IAEA, Vienna) Vol. III, $P$. 419. Ulrickson, M. et al. (1990). J. Nucl. Mater. 126/212, 44.

waltz, R. E. et al. (1990a). Proceedings of the Thirteenth International Conference on Plasma Physics and Controlled Nuclear Eusion Research, Washington, 1990, (IAEA, Vienna) Paper IAEA-CN-53/D-4-7, to appear.

Waltz, R. E., DeBoo, J. C., and Rosenbluth, M. N. (1990b). Phys. Rev. Lett. 65, 2390 .

Nong, K. L. et al. (1991a). Phys. Rev. Lett. 66, 1874,

Wong, K. L. et al. (1991b). At this conference.

Wooteon, A. J. et al. (1990). Phys. Flulds B 2, 2879 .

Yushmanov, P. et al. (1990). Nucl. Eusion 25, 65.

zarnstorff, M. C. et al. (1987). Controlled Eusion and Plasma Physics, Proceedings of the Fourteenth European Conference, Madrid, 1987. Vol. 110, Part 1, P. 144.

Zarnstorff, M. C. et al. (1988a). Phys. Rev. Lett. 60, 1306.

zarngtorff, M. C. ot al. (1988b). Controlled Fusion and Plasma Heating, Proceedings of the Fifteenth European Conference, Dubrovnik, 1988. Vol. 128, Part 1, p. 95 .

Zarngtorff, M. C. et al. (1989a). Plasma Phys. Control. Nucl. Fus. Res., Proceedings of the Twelfth International Conf., Nice, 1988 , (IAEA, Vienna) Vol. I, p. 183.

zazngtozff. M. C. et al (1989b). Controlled Fusion and Plasma Physics, Proceedings of the Sixteenth European Conference, Venice 1989. Vol. 13B, Part 1, p. 3.5.

zarnstorff, M. C. et al. (1990a). Phys. Fluids B $2,1852$.

zarnstorff, M. C. et al. (1990b). Proceedings of the Thirteenth International Confezence on Plasma Physics and Controlled Nuclear Fusion Research, washington, 1990 (IAEA, Vienna) Paper IAEA-CN-53/A-II-2, to appear.

Zarnstorff, M. C. et al. $(1990 \mathrm{C})$. Colltrolled Fusion and Plasma Heating, Proceedings of the Seventeenth European Conference, Amsterdam, 1990. Vo1. 14B, Part 1, p. 42. zweben, S. J., iad Medley, S. S. (1989). Phys. Fluids B $1,2058$.

Zweben, S. J. et al. (1990). Nucl. Eusion 30, 1551.

zweben, S. J. et a.. (1991). Prince:on Plasma Physics Laboratory Report PPPL-2770. 
Dr. F. Paoloni. Univ. of Wollongong, AUSTRALIA

Prot. M.H. Brennan, Univ. of Sydney, AUSTRALIA

Plasma Research Lab., Australian Nat. Univ., AUSTRALIA

Prot. I.R. Jones. Flindors Univ. AUSTRALIA

Prof. F. Cap. Inst. for Theoretical Physics. AUSTRIA

Prof. M. Heindler. Institut fur Theoretische Physik. AUSTRIA

Prof. M. Goossans. Astronomisch Instituut, BELGIUM

Ecole Royale Militairs, Lao. Phy. Plasmas, BELGIUM

Commission-European, DG. XII-Fusion Prog.. BÉLGIUM

Prof R. Bouciaive, Rijksuniversitert Gent. BELGIUM

Dr. P.H. Sakanaka, Instituto Fisica, BPAZIL

Instuuto De Pesquisas Espaciais-INPE, BRAZIL

Documents Oftice. Alomic Energy of Canada Lid. CANADA

Dr. M.P. Bachynski. MPB Technologies, Inc., CANADA

Dr. H.M. Skarsgard. Univ. of Saskatchewan, CANADA

Prof. J. Teichmann. Univ. of Montreal, CANADA

Prof. S.R. Sreenivasan. Univ. of Caigary, CANADA

Prof. T.W. Johnston, INAS-Energie, CANADA

Dr. R. Botton, Centre canadien de husion magnévque, CANADA

Dr. C.R. James.. Univ. of Alberta. CANADA

Dr. P. Lukac, Komenskeho Universzita. CZECHOSLOVAKIA

The Librarian, Cuiham Laboratory, ENGLAND

Library, R61, Ruthertord Appleton Laboratory, ENGLAND

Mrs. S.A. Hutchunson, JET Library. ENGLAND

P Măhơnen, Univ of Helsinki. FINLAND

C. Mountet, Lab de Physique des Milieux lonises. FRANCE

J. Radel, CEN/CADARACHE - Bat 506, FRANCE

Ms. C. Rinni. Univ. of loannina, GREECE

Dr. T. Mual, Acaderny Bibliographic Ser., HONG KONG

Preprnt Library. Hungarian ACademy of Sa., HUNGARY

Dr. B. Das Gupta. Saha inst. of Nudear Physics. INDIA

Dr. P. Kar: Inst. for Plasma Research, INDIA

Dr. P. Rosenau, Israel inst of Tochnology, ISPAEL

Libranan, Internatonal Center for Theo Physics, ITALY

Miss C. Do Palo. Associazione EURATOM-ENEA, ITALY

Dr. G. Grosso, Isstuto di Fisica del Plasma, ITALY

Dr. H Yamato. Toshiba Res \& Dovel Cinter. JAPAN

Prof. I. Kawakami, Atomic Enorgy Res. Inst., JAPAN

Prot. K. Nishikawa. Hiroshima Univ. JAPAN
Director, Japen Atomic Energy Research Inst.. JAPAN

Prot. S. Itoh. Kyushu Univ., JAPAN

Data and Planning Center, Nagoya Univ. JAPAN

Prof. S. Tanaka. Kyoto Univ., JAPAN

Library. Kyoto Univ., JAPAN

Prof. N. Inove, Univ. of Tokyo. JAPAN

S. Mon, Technical Advisor, JAERI, JAPAN

O. Mitara, Kumamoto Inst. of Tochnology. JAPAN

H. Jeong. Kores Advanced Energy Research Inst., KOREA

Prot. D.I. Choi. The Korea Adv. Inst. of Sa. \& Tech., KOREA

Prof. B.S. Liloy, Univ. of Waikato. NEW ZEALAND

Inst. of Plasma Physics. PEOPLE'S REPUBLIC OF CHINA

Librarian, Inst. of Physics, PEOPLE'S REPUBUC OF CHINA

Library. Tsinghua Univ., PEOPLE'S REPUBLIC OF CHINA

2. L. S.W. Inst Physics, PEOPLE'S REPUBLC OF CHINA

Prof. J.A.C. Cabral, Insututo Supenor Tecrico, PORTUGAL

Dr. O. Pegrus, ALI CUZA Univ., ROMANIA

Dr. J. de Villiers, Fusion Studios, AEC, S. AFRICA

Prot. M.A. Hellberg, Univ. of Natal, S. AFRICA

C.I.E.M.A.T. Fusion Division Library, SPAIN

Dr. L Stantlo, Univ. of UMEA, SWEDEN

Library, Roya Inst. of Tectinology, SWEDEN

Prot. H. Wilheimson, Chaimers Univ. of Tech., SWEDEN

Centre Phys. Des Plasmas. Ecole Polytoch. SWITZERLAND

Bibliothok, Inst. Voor Plasma-Fysica. THE NETHEPLANDS

M. Durgut, Vice Charman, Middle East Tech. Univ., TURKE

Dr. D.D. Ryutov. Siberian Branch of Academy of Sci. USSR

Dr. G.A. Eliseevv. Kurchatov Inst. USSR

Libranan. The Ukr.SSR Academy of Scionces. USSR

Dr. L.M. Kovnzhnykh, Inst of General Physics, USSR

Kemforschungsaniage GmbH. Zentrabibliothek, W. GERMAI

Bibtiothek, Inst. Für Plasmatorschung, W. GERMANY

Prof. K. Schinder, Ruhr-Universital Bochum, W. LERMANY

Dr. F. Wagner, (ASDEX), Max-Planck-Instutu, W GERMANY Libranan, Max-Planck-insotut, W GERMANY

Prof. R.K. Janev, Inst. of Physics, YUGOSLAVIA 

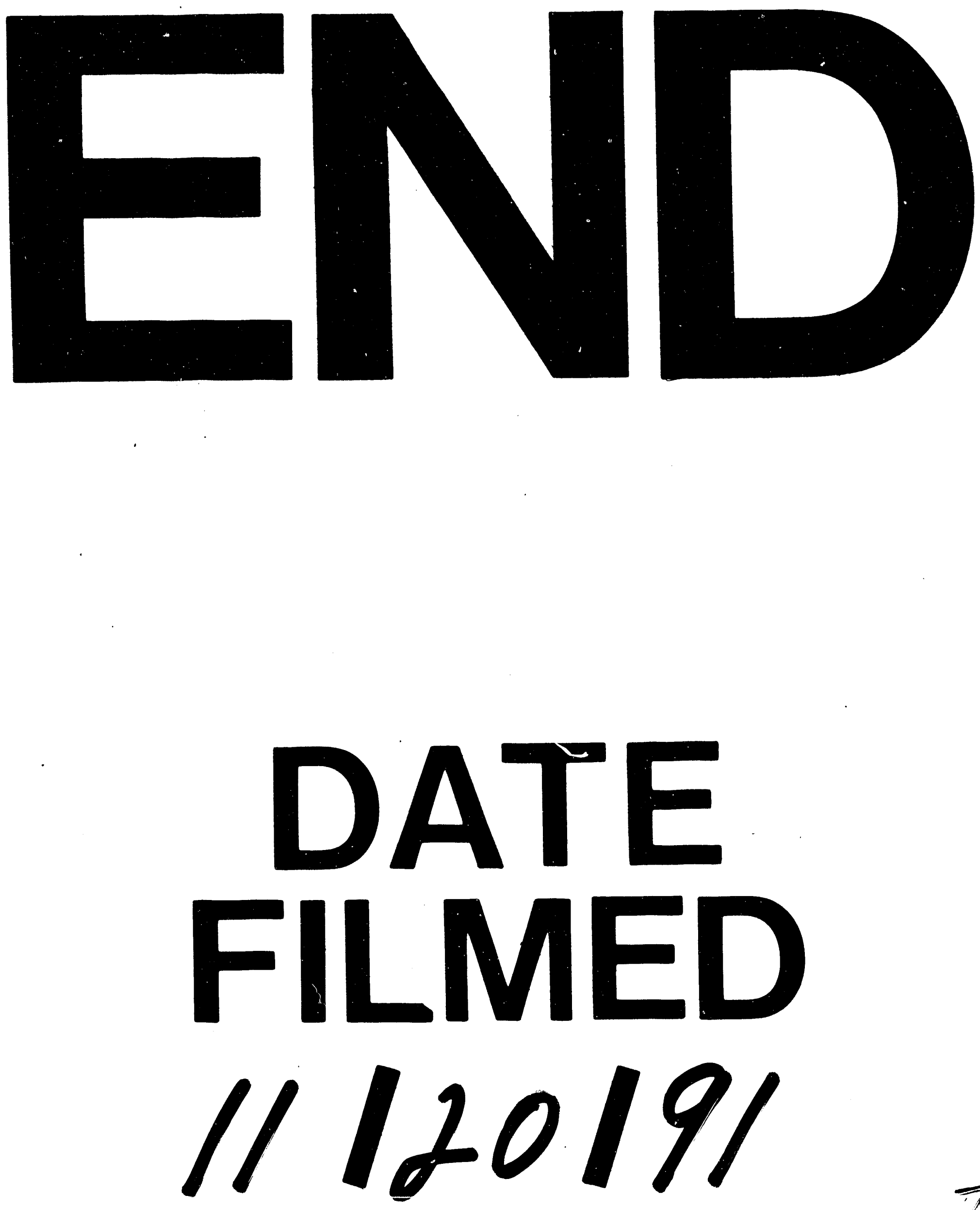

II 
